\title{
Numerical Methods for Optimal Dividend Payment and Investment Strategies of Regime-switching Jump Diffusion Models with Capital Injections
}

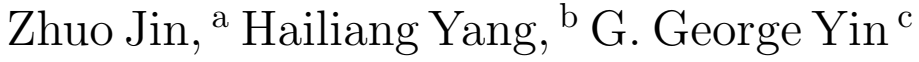 \\ ${ }^{a}$ Centre for Actuarial Studies, Department of Economics, The University of Melbourne, VIC 3010, Australia, \\ zjin@unimelb.edu.au. This research was supported in part by Early Career Research Grant by The University of Melbourne. \\ ${ }^{\mathrm{b}}$ Department of Statistics and Actuarial Science, The University of Hong Kong, Hong Kong. Email: hlyang@hkusua.hku.hk. \\ The research of this author was supported in part by Research Grants Council of HKSAR (Project No: HKU 706611P). \\ ${ }^{\mathrm{c}}$ Department of Mathematics, Wayne State University, Detroit, Michigan 48202, gyin@math.wayne.edu. The research of this \\ author was supported in part by the National Science Foundation under DMS-120766\%.
}

\begin{abstract}
This work focuses on numerical methods for finding optimal investment, dividend payment, and capital injection policies to maximize the present value of the difference between the cumulative dividend payment and the possible capital injections. The surplus is modeled by a regime-switching jump diffusion process subject to both regular and singular controls. Using dynamic programming principle, the value function is a solution of coupled system of nonlinear integro-differential quasivariational inequalities. In this paper, the state constraint of the impulsive control gives rise to a capital injection region with free boundary, which makes the problem even more difficult to analyze. Together with the regular control and regimeswitching, the closed-form solutions are virtually impossible to obtain. We use Markov chain approximation techniques to construct a discrete-time controlled Markov chain to approximate the value function and optimal controls. Convergence of the approximation algorithms is proved. Examples are presented to illustrate the applicability of the numerical methods.
\end{abstract}

Key words: Stochastic control, singular control, investment strategy, dividend policy, capital injection, free boundary, Markov chain approximation.

\section{Introduction}

Designing dividend payment policies has long been an important issue in finance and actuarial sciences. Because of the nature of their products, insurers tend to accumulate relatively large amounts of cash, cash equivalents, and investments in order to pay future claims and avoid insolvency. The payment of dividends to shareholders may reduce an insurer's ability to survive adverse investment and underwriting experience. A practitioner will manage the reserve and dividend payment against asset risks so that the company can satisfy its minimum capital requirement.

Stochastic optimal control problems on dividend strategies for an insurance corporation have drawn increasing attention since the introduction of the optimal dividend payment model proposed by [De Finetti, 1957]. There have been increasing efforts on using advanced methods from the toolbox of stochastic control to study the optimal dividend policy; see [Asmussen and Taksar, 1997], [Yin et al., 2010], and [Jin et al., 2012]. Browne studied the optimal investment strategy for a firm with the constraint of probability of ruin in [Browne, 1995]. [Azcue and Muler, 2010] analyzed the problem of the maximization of total discounted dividend payment for an insurance company. Empirical studies indicate, in particular, that traditional surplus models fail to capture more extreme movements such as market switching. To reflect reality, much effort has been devoted to produce better models. One of the recent trends is to use regime-switching models. [Hamilton, 1989] introduced a regime-switching time series model. Recent work on risk models and related issues can be found in [Yang and Yin, 2004]. Optimal dividend strategies were studied in a regime-switching diffusion model in [Sotomayor and Cadenillas, 2011]. 
To maximize the expected total discounted dividend payments, the company will bankrupt almost surely if the dividend payment is paid out as a barrier strategy. In practice, [Dickson and Waters, 2004] suggested that capital injections can be taken into account to avoid insolvency when capital reserve is insufficient. Furthermore, transaction cost will be considered; see also [Sethi and Taksar, 2002], [Kulenko and Schimidli, 2008], and [Yao et al., 2011]. Whenever the company is on the verge of financial ruin, the company has the opportunity to raise sufficient funds to survive. A natural payoff function is maximizing the difference between the expected total discounted dividend payment and the capital injections with costs until bankruptcy under the optimal controls.

In this work, we aim to obtain the optimal dividend payment and investment strategies using the collective risk model under the Makovian regime-switching setting with capital injections. We allow the investment of surplus in a continuous-time financial market and the management of the dividend payment policy. In our model, borrowing money to do risky investment is not allowed. The insurers cannot put too much money in risky assets for the sake of risk management. That is, there is a natural constraint on the portfolio so that the total weight of the risky assets should be no more than 1 . Another constraint on the investment is that short selling risky asset is prohibited. Hence, the proportion of capital invested in the risky asset is denoted as a regular control $u \in[0,1]$. In addition, a dividend process is not necessarily absolutely continuous. In fact, dividends are usually paid out at random discrete times, where insurance companies may distribute dividends at unrestricted payment rate. In such a scenario, the surplus level changes drastically on a dividend payday. Thus, abrupt or discontinuous changes occur due to "singular" dividend distribution policy. Moreover, the capital injections, modeled by impulse controls are exerted when surplus hits not only 0 but also a sufficiently low threshold. To maximize the performance, the impulse controls of capital injections depend on the surplus processes and can be very large, which results in a free boundary of capital injection region and adds more difficulty to analyze the optimal policies. Together with the Markov switching and the incurred claims, this gives rise to a regime-switching jump diffusion stochastic control problem with singular and impulse controls.

To find the optimal strategies, one usually solves a so-called Hamilton-Jacobi-Bellman equation. However, because of the regime-switching jump diffusion and the mixed regular and singular control formulation, the Hamilton-Jacobi-Bellman equation is in fact a coupled system of nonlinear integro-differential quasi-variational inequalities. A closed-form solution is virtually impossible to obtain. A viable alternative is to employ numerical approximations. In this work, we adopt the Markov chain approximation methodology developed in [Kushner and Dupuis, 2001]. To the best of our knowledge, numerical methods for capital injections of regime-switching jump diffusions have not been studied in the literature to date. [Kushner and Martins, 1991] and [Budhiraja and Ross, 2007] considered singular controlled diffusions without regime switching. We focus on developing numerical methods that are applicable to find optimal controls for regime-switching jump diffusion models. A numerical algorithm for approximating optimal reinsurance and dividend payment policies under regime-switching diffusion models was developed in [Jin et al., 2012]. In this project, we analyze the numerical algorithm of investment strategy and dividend payment policy under a regime-switching formulation, and carried out a convergence analysis using weak convergence and relaxed control formulation of numerical schemes for singular control problems in the setting of regime switching, in which case one needs to deal with a system of quasi-variational inequalities. This paper further treats models with capital injections. As a result, we have to deal with impulse controls. Roughly speaking, due to the singular and impulse controls, the value function in each regime is verified to be a concave function and defined separately in three regions, which are capital injection region, continuation region, and dividend payment region. Taking into consideration of capital injections, the capital injections have to be ordered if the surplus violates the capital requirement for running the business. Hence, the impulse controls of capital injections will occur for sure at zero surplus. In addition, the optimization of payoff function will lead the barrier of capital injection region to be a free boundary. Thus, the impulse controls of capital injections depend on the surplus process and can be very large. These state-dependent capital injections lead to the formulation of free boundary problem, and the state-dependent "threshold" curve, as demonstrated in the numerical experiments, separates the capital injection region and continuation region. Due to the complexity of the construction, closed-form solutions are virtually impossible to be obtained and the numerical scheme thus is a viable alternative. We construct the feasible numerical approximation schemes for finding a good approximation to the underlying problems. It is worth mentioning that the Markov chain approximation method requires little regularity of the value function and/or analytic properties of the associated systems of Hamilton-JacobiBellman equations, or quasi-variational inequalities, or integro-differential quasi-variational inequalities. The numerical implementation can be done using either value iterations or policy iterations.

The rest of the paper is organized as follows. A general formulation of optimal investment strategy, dividend policies, capital injections and assumptions are presented in Section 2. Certain properties of the optimal value function and the verification theorem are also presented. Section 3 deals with the numerical algorithm of Markov chain approximation method. The Poisson 
jumps, regular control, the singular and impulse control are well approximated by the approximating Markov chain and the dynamic programming equations are presented. Section 4 deals with the convergence of the approximation scheme. The technique of "rescaling time" is introduced and the convergence theorems are proved. Three numerical examples are provided in Section 5 to illustrate the performance of the approximation method. Finally, additional remarks are provided in Section 6 .

\section{Formulation}

The surplus process $X(t)$ under consideration is a jump diffusion process with regime-switching under singular and impulse control. To delineate the random environment and other random factors, we use a continuoustime Markov chain $\alpha(t)$ taking values in the finite space $\mathcal{M}=\left\{1, \ldots, n_{0}\right\}$. For each $i \in \mathcal{M}$, the premium rate is $c(i)>0$. Let $\varphi_{n}$ be the inter-arrival time of the $n$th claim, $\nu_{n}=\sum_{j=1}^{n} \varphi_{n}$. For a slightly more generality, we consider a Poisson measure in lieu of the traditionally used Poisson process. Suppose $\Gamma \subset \mathbb{R}_{+}$is a compact set and the function $q(X, i, \rho)$ is the magnitude of the claim sizes, where $\rho$ has distribution $\Pi(\cdot) . N(t, H)=$ number of claims on $[0, t]$ with claim size taking values in $H \in \Gamma$. Note that our formulation is general, the claim sizes are assumed to depend on the switching regime. Then the Poisson measure $N(\cdot)$ has intensity $\lambda d t \times \Pi(d \rho)$ where $\Pi(d \rho)=f(\rho) d \rho$. Assume that $q(\cdot, i, \rho)$ is continuous for each $\rho$ and each $i \in \mathcal{M}$. At different regimes, the values of $q(\cdot, i, \rho)$ could be much different, which takes into consideration of random environment. Then the surplus process in the absence of dividend payment and investment is a regime-switching jump process given by

$$
\begin{aligned}
d \tilde{X}(t) & =\sum_{i \in \mathcal{M}} I_{\{\alpha(t)=i\}}(c(i) d t-d R(t)) \\
& =c(\alpha(t)) d t-\int_{\Gamma} q\left(X\left(t^{-}\right), \alpha(t), \rho\right) N(d t, d \rho),
\end{aligned}
$$

where

$$
R(t)=\int_{0}^{t} \int_{\Gamma} q\left(X\left(s^{-}\right), \alpha(s), \rho\right) N(d s, d \rho) .
$$

We consider the financial market with a risk free asset $Y(t)$ and a risky asset $S(t)$ with prices satisfying

$$
\left\{\begin{array}{l}
\frac{d Y(t)}{Y(t)}=g(\alpha(t)) d t \\
\frac{d S(t)}{S(t)}=b(\alpha(t)) d t+\sigma(\alpha(t)) d W(t)
\end{array}\right.
$$

where for each $i \in \mathcal{M}, g(i)$ and $b(i)$ are the return rates of the risk free and risky asset, respectively. $\sigma(\alpha(t))$ is the corresponding volatility and $W(t)$ is a standard Brownian motion. The investment behavior of the insurer is modelled as a portfolio process $u(t)$, where proportional surplus $u(t) \in[0,1]$ was invested in the risky asset $S(t)$. We are now working on a filtered probability space $\left(\Omega, \mathcal{F},\left\{\mathcal{F}_{t}\right\}, P\right)$, where $\mathcal{F}_{t}$ is the $\sigma$-algebra generated by $\{\alpha(s), W(s), N(s): 0 \leq s \leq t\}$.

A dividend strategy $z(\cdot)$ is an $\mathcal{F}_{t}$-adapted process $\{z(t)$ : $t \geq 0\}$ corresponding to the accumulated amount of dividends paid up to time $t$ such that $z(t)$ is a nonnegative and nondecreasing stochastic process that is right continuous with left limits. Throughout the paper, we use the convention that $z\left(0^{-}\right)=0$.

Remark 1 Note that $b$ describes the yield rate of the risky assets, and is modulated by a finite-state Markov chain $\alpha(t)$, which represents the market mode and describes the economics impact over a long time period that cannot be modeled as a classical differential equation. It is used to determine the yield rate of the financial assets and mainly depends on the market, not surplus. Like the yield rate $b$, the premium rate $c$ and volatility $\sigma$ are mainly affected by the market mode. From a numerical approximation point of view, making $c, b$ and $\sigma$ $X$-dependent will not introduce any essential difficulty.

The capital injection process $l(t)=\sum_{n=1}^{\infty} I_{\left\{\tau_{n} \leq t\right\}} \zeta_{n}$ is described by a sequence of increasing stopping times $\left\{\tau_{n}, n=1,2, \ldots\right\}$ and a sequence of random variables $\left\{\zeta_{n}, n=1,2, \ldots\right\}$, which represent the times and the sizes of capital injections. A control policy $\pi$ is described by $\pi=\{u, z ; l\}=\left\{u, z ; \tau_{1}, \ldots, \tau_{n}, \ldots ; \zeta_{1}, \ldots, \zeta_{n}, \ldots\right\}$. Assume the evolution of $X(t)$, subject to capital injections and dividend payments, follows a one-dimensional process on an unbounded domain $G^{\prime}=(0, \infty)$. The surplus process considers dividend payment, capital injection and investment satisfy the following stochastic differential equation

$$
\left\{\begin{aligned}
X(t) & =x+\int[[g(\alpha(t))(1-u(t))+u(t) b(\alpha(t))] X(t) \\
& +c(\alpha(t))] d t+\int u(t) \sigma(\alpha(t)) X(t) d W(t)-R(t) \\
& -z(t)+\sum_{n=1}^{\infty} I_{\left\{\tau_{n} \leq t\right\}} \zeta_{n}, \\
X(0) & =x
\end{aligned}\right.
$$

for all $t<\tau$ and we impose $X(t)=0$ for all $t>\tau$, where $\tau=\inf \{t \geq 0: X(t) \leq 0\}$ represents the time of ruin. The jump size of $z$ at time $t \geq 0$ is denoted by $\Delta z(t):=$ $z(t)-z\left(t^{-}\right)$, and $z^{c}(t):=z(t)-\sum_{0 \leq s \leq t} \Delta z(s)$ denotes the continuous part of $z$. Also note that $\Delta X(t):=X(t)-$ $X\left(t^{-}\right)=-\Delta z(t)$ for any $t \geq 0$.

In this paper, we assume that the shareholders can get the proportion of $\beta_{1}$ for every dividend payment, where $0<\beta_{1}<1$. We omit the fixed transaction costs in the dividends payout process. Moreover, we assume that the 
shareholders need to pay $K+\beta_{2} \zeta, \beta_{2}>1$, to meet the capital injection of $\zeta . K>0$ is the fixed transaction costs, $\left(\beta_{2}-1\right) \zeta$ is the proportional transaction costs. Denote by $r>0$ the discount factor. For an arbitrary admissible pair $\pi=(u, z, l)$, the performance function is

$$
\begin{aligned}
J(x, i, \pi)= & E_{x, i}\left[\int_{0}^{\infty} e^{-r t} \beta_{1} d z-\sum_{n=1}^{\infty} e^{-r \tau_{n}}\left(K+\beta_{2} \zeta_{n}\right)\right. \\
& \left.\times I_{\left\{\tau_{n}<\infty\right\}}\right] .
\end{aligned}
$$

The pair $\pi=(u, z, l)$ is said to be admissible if $u, z$, and $l$ satisfy

(i) $u(t), z(t)$, and $l(t)$ are nonnegative for any $t \geq 0$,

(ii) $z$ is right continuous, has left limits, and is nondecreasing,

(iii) $X(t) \geq 0$, for any $t \leq \tau$,

(iv) $u, z$ and $l$ are adapted to $\mathcal{F}_{t}:=\sigma\{\alpha(s), W(s), N(s)$, $0 \leq s \leq t\}$ augmented by the $P$-null sets,

(v) $\tau_{n}$ is a stopping time w.r.t. $\mathcal{F}_{t}$, and $0 \leq \tau_{1}<\cdots<$ $\tau_{n}<\cdots$, a.s.

(vi) $\zeta_{n}$ is measurable w.r.t. $\mathcal{F}_{t}$

(vii) $P\left(\lim _{n \rightarrow \infty} \tau_{n}<T\right)=0, \forall T>0$, and

(viii) $J(x, i, \pi)<\infty$ for any $(x, i) \in G \times \mathcal{M}$ and admissible pair $\pi=(u, z, l)$, where $J$ is the functional defined in (2.4).

Suppose that $\mathcal{A}$ is the collection of all admissible pairs. Define the value function as

$$
V(x, i):=\sup _{\pi \in \mathcal{A}} J(x, i, \pi)
$$

For an arbitrary $\pi \in \mathcal{A}, i=\alpha(t) \in \mathcal{M}$, and $V(\cdot, i) \in$ $C^{2}(\mathbb{R})$, define an operator $\mathcal{L}^{\pi}$ by

$$
\begin{aligned}
\mathcal{L}^{\pi} V(x, i) & =V_{x}(x, i)([g(i)(1-u)+u b(i)] x+c(i)) \\
& +\frac{1}{2} \sigma(i)^{2} u^{2} x^{2} V_{x x}(x, i) \\
& +\lambda \int_{0}^{\infty}[V(x-q(x, i, \rho), i)-V(x, i)] f(\rho) d \rho \\
& +Q V(x, \cdot)(i),
\end{aligned}
$$

where $V_{x}$ and $V_{x x}$ denote the first and second derivatives with respect to $x$, and

$$
Q V(x, \cdot)(i)=\sum_{j \neq i} q_{i j}(V(x, j)-V(x, i))
$$

Define another capital injection operator $\mathcal{H}$ by

$$
\mathcal{H} V(x, i)=\sup _{\widetilde{y} \geq 0}\left\{V(x+\widetilde{y}, i)-\beta_{2} \widetilde{y}-K\right\}
$$

If the value function $V$ defined in (2.5) is sufficiently smooth, by applying the dynamic programming principle ([Fleming and Soner, 2006]), $V$ formally satisfies the following quasi-variational inequalities (QVIs):

$$
\begin{aligned}
& \max \left\{\mathcal{L}^{\pi} V(x, i)-r V(x, i), \beta_{1}-V_{x}(x, i)\right. \\
& \mathcal{H} V(x, i)-V(x, i)\}=0, \quad \text { for each } i \in \mathcal{M}
\end{aligned}
$$

Similar to [Yao et al., 2011], we divide the set of the surplus to three regions

(i) Continuation region:

$$
\begin{gathered}
\mathcal{C}:=\left\{\mathcal{L}^{\pi} V(x, i)-r V(x, i)=0, \beta_{1}<V_{x}(x, i),\right. \\
\mathcal{H} V(x, i)<V(x, i)\}
\end{gathered}
$$

(ii) Dividend payout region:

$$
\begin{gathered}
\mathcal{D}:=\left\{\mathcal{L}^{\pi} V(x, i)-r V(x, i)<0, \beta_{1}=V_{x}(x, i),\right. \\
\mathcal{H} V(x, i)<V(x, i)\}
\end{gathered}
$$

(iii) Capital injection region:

$$
\begin{gathered}
\mathcal{I}:=\left\{\mathcal{L}^{\pi} V(x, i)-r V(x, i)<0, \beta_{1}<V_{x}(x, i),\right. \\
\mathcal{H} V(x, i)=V(x, i)\} .
\end{gathered}
$$

Boundary Conditions. The capital injection will be taken into account when there is not enough solvency capital to maintain the business. Intuitively, for all $i \in$ $\mathcal{M}$, on the boundary of the capital injection region, the value function obeys

$$
V(x, i)=\underset{\widetilde{y} \geq 0}{\sup }\left\{V(x+\widetilde{y}, i)-\beta_{2} \widetilde{y}-K\right\}
$$

To make the company run continuously, the capital injections will definitely occur at the moments when $x=0$. In addition, the capital injections also occur whenever the surplus is sufficiently low. The impulse control of capital injections is dependent on the surplus states and leads to a free boundary of the capital injection region. Furthermore, we also need boundary conditions when $x \rightarrow \infty$. Since the surplus cannot reach infinity, we only need to choose $B$ large enough and compute the value function in the finite interval $G=[0, B]$. To make it computationally feasible, we truncate $x$ at some large value $B$. When $B$ is large enough, it follows

$$
V_{x}(B, i)=\beta_{1}
$$

That is, the dividend payout strategy is a barrier strategy. Whenever the surplus exceeds certain barrier, the excess is paid out immediately as dividend. 
We consider the dividend payout strategy with the capital injection as a band strategy. The decision maker will take no action until the surplus reaches the lower barrier, where a impulse control of capital injection will be taken. The dividend will be paid out immediately when the surplus reaches the upper barrier. Combining (2.8), (2.9) and (2.10), the system of QVIs with the boundary conditions is given by

$$
\left\{\begin{array}{l}
\max \left\{\mathcal{L}^{\pi} V(x, i)-r V(x, i),\right. \\
\left.\quad \beta_{1}-V_{x}(x, i), \mathcal{H} V(x, i)-V(x, i)\right\}=0, i \in \mathcal{M}, \\
V_{x}(B, i)=\beta_{1}, \\
V(0, i)=\sup _{0 \leq \widetilde{y} \leq B}\left\{V(\widetilde{y}, i)-\beta_{2} \widetilde{y}-K\right\} .
\end{array}\right.
$$

\section{Numerical Algorithm}

Our goal is to design a numerical scheme to approximate value function $V$ in (2.5). As a standard assumption, we assume $V(\cdot)$ is continuous with respect to $x$. In this section we construct a locally consistent Markov chain approximation for the jump diffusion model with singular control and regime-switching. The discrete-time and finite-state controlled Markov chain is so defined that it is locally consistent with (2.3). First let us recall some facts of Poisson random measure which is useful for constructing the approximating Markov chain and for the convergence theorem.

There is an equivalent way to define the process (2.3) by working with the claim times and values. To do this, set $\nu_{0}=0$, and let $\nu_{n}, n \geq 1$, denote the time of the $n$th claim, and $q\left(\cdot, \cdot, \rho_{n}\right)$ is the corresponding claim intensity with a suitable function of $q(\cdot)$. Let $\left\{\nu_{n+1}-\right.$ $\left.\nu_{n}, \rho_{n}, n<\infty\right\}$ be mutually independent random variables with $\nu_{n+1}-\nu_{n}$ being exponentially distributed with mean $1 / \lambda$, and let $\rho_{n}$ have a distribution $\Pi(\cdot)$. Furthermore, let $\left\{\nu_{k+1}-\nu_{k}, \rho_{k}, k \geq n\right\}$ be independent of $\left\{x(s), \alpha(s), s<\nu_{n}, \nu_{k+1}-\nu_{k}, \rho_{k}, k<n\right\}$, then the $n$th claim term is $q\left(X\left(\nu_{n}^{-}\right), \alpha\left(\nu_{n}\right), \rho_{n}\right)$, and the claim amount $R(t)$ can be written as

$$
R(t)=\sum_{\nu_{n} \leq t} q\left(X\left(\nu_{n}^{-}\right), \alpha\left(\nu_{n}\right), \rho_{n}\right)
$$

We note the local properties of claims for (2.3). Because $\nu_{n+1}-\nu_{n}$ is exponentially distributed, we can write

$$
\begin{gathered}
P\{\text { claim occurs on }[t, t+\delta) \mid x(s), \alpha(s), W(s), \\
N(s, \cdot), s \leq t\}=\lambda \delta+o(\delta) .
\end{gathered}
$$

By the independence and the definition of $\rho_{n}$, for any $H \in \mathcal{B}(\Gamma)$, we have

$$
\begin{aligned}
& P\left\{X(t)-X\left(t^{-}\right) \in H \mid t=\nu_{n} \text { for some } \mathrm{n} ;\right. \\
& \left.\quad W(s), X(s), \alpha(s), N(s, \cdot), s<t ; X\left(t^{-}\right)=x, \alpha(t)=\alpha\right\} \\
& \quad=\Pi\left(\rho: q\left(X\left(t^{-}\right), \alpha(t), \rho\right) \in H\right) .
\end{aligned}
$$

It is implied by the above discussion that $x(\cdot)$ satisfying (2.3) can be viewed as a process that involves regimeswitching diffusion with claims according to the claim rate defined by (3.1). Given that the $n$th claim occurs at time $\nu_{n}$, we construct the values according to the conditional probability law (3.2) or, equivalently, write it as $q\left(X\left(\nu_{n}^{-}\right), \alpha\left(\nu_{n}\right), \rho_{n}\right)$. Then the process given in (2.3) is a switching diffusion process until the time of the next claim. To begin, we construct a discrete-time, finitestate, controlled Markov chain to approximate the controlled diffusion process with regime-switching, and the dynamic system is given by

$$
\left\{\begin{aligned}
d X(t) & =[[g(\alpha(t))(1-u(t))+u(t) b(\alpha(t))] X(t) \\
& +c(\alpha(t))] d t+u(t) \sigma(\alpha(t)) X(t) d W(t), \\
X(0) & =x
\end{aligned}\right.
$$

\subsection{Approximating Markov Chain}

We will construct a locally consistent Markov chain approximation for the mixed regular-singular control regime-switching jump diffusion model with impulse controls. The discrete-time controlled Markov chain is so defined that it is locally consistent with (2.3). Note that the state of the process has two components $x$ and $\alpha$. Hence in order to use the methodology in [Kushner and Dupuis, 2001], our approximating Markov chain must have two components: one component delineates the diffusive behavior whereas the other keeps track of the regimes. Let $h>0$ be a discretization parameter representing the step size. Define $S_{h}^{\prime}=\{x: x=k h, k=0, \pm 1, \pm 2, \ldots\}$ and $S_{h}=S_{h}^{\prime} \cap G_{h}$, where $G_{h}=(0, B+h)$ and $B$ is an upper bound introduced for numerical computation purpose. Moreover, assume without loss of generality that the boundary point $B$ is an integer multiple of $h$. Let $\left\{\left(\xi_{n}^{h}, \alpha_{n}^{h}\right), n<\infty\right\}$ be a controlled discrete-time Markov chain on $S_{h} \times \mathcal{M}$ and denote by $p_{D}^{h}\left((x, i),(y, j) \mid \pi^{h}\right)$ the transition probability from a state $(x, i)$ to another state $(y, j)$ under the control $\pi^{h}$. We need to define $p_{D}^{h}$ so that the chain's evolution well approximates the local behavior of the controlled regime-switching diffusion (3.3). At any discrete time $n$, we can either exercise a regular control, a singular control or a impulse control step. That is, if we 
put $\Delta \xi_{n}^{h}=\xi_{n+1}^{h}-\xi_{n}^{h}$, then

$$
\begin{aligned}
\Delta \xi_{n}^{h}= & \Delta \xi_{n}^{h} I_{\{\text {capital injection at } n\}} \\
& +\Delta \xi_{n}^{h} I_{\{\text {dividend payment at } n\}} \\
& +\Delta \xi_{n}^{h} I_{\{\text {regular control at } n\}} .
\end{aligned}
$$

The chain and the control will be chosen so that there is exactly one term in (3.4) is nonzero. Denote by $\left\{I_{n}^{h}: n=0,1, \ldots\right\}$ a sequence of control actions, where $I_{n}^{h}=0,1$ or 2 , if we exercise a capital injection, dividend payment, or regular control at time $n$, respectively.

If $I_{n}^{h}=0$, then we denote by $\Delta l_{n}^{h}$ the impulse control that is the capital injection for the chain at time $n$. Note that $\Delta \xi_{n}^{h}=\Delta l_{n}^{h}$. If $I_{n}^{h}=1$, or $\xi_{n}^{h}=B+h$, dividend payment is exerted definitely as a singular control. Dividend is paid out to lower the surplus level. Moreover, we require this dividend payment takes the state from $B+h$ to $B$. That is, if we denote by $\Delta z_{n}^{h}$ the random variable that is the impulse control for the chain at time $n$, then $\Delta \xi_{n}^{h}=-\Delta z_{n}^{h}=-h$.

If $I_{n}^{h}=2$, then we denote by $u_{n}^{h} \subset U$ the random variable that is the regular control action for the chain at time $n$. Let $\tilde{\Delta} t^{h}(\cdot, \cdot, \cdot)>0$ be the interpolation interval on $S_{h} \times \mathcal{M} \times U$. Assume $\inf _{x, i, u} \tilde{\Delta} t^{h}(x, i, u)>0$ for each $h>0$ and $\lim _{h \rightarrow 0} \sup _{x, i, u} \tilde{\Delta} t^{h}(x, i, u) \rightarrow 0$.

Let $E_{x, i, n}^{u, h, 2}, \operatorname{Var}_{x, i, n}^{u, h, 2}$ and $P_{x, i, n}^{u, h, 2}$ denote the conditional expectation, variance, and marginal probability given $\left\{\xi_{k}^{h}, \alpha_{k}^{h}, u_{k}^{h}, I_{k}^{h}, k \leq n, \xi_{n}^{h}=x, \alpha_{n}^{h}=i, I_{n}^{h}=2, u_{n}^{h}=u\right\}$, respectively. The sequence $\left\{\left(\xi_{n}^{h}, \alpha_{n}^{h}\right)\right\}$ is said to be locally consistent, if it satisfies

$$
\begin{aligned}
& E_{x, i, n}^{u, h, 2}\left[\Delta \xi_{n}^{h}\right]=[[g(i)(1-u)+u b(i)] x+c(i)] \tilde{\Delta} t^{h}(x, i, u) \\
& +o\left(\tilde{\Delta} t^{h}(x, i, u)\right) \text {, } \\
& \operatorname{Var}_{x, i, n}^{u, h, 2}\left(\Delta \xi_{n}^{h}\right)=u^{2} \sigma^{2}(i) x^{2} \tilde{\Delta} t^{h}(x, i, u)+o\left(\tilde{\Delta} t^{h}(x, i, u)\right), \\
& P_{x, i, n}^{u, h, 2}\left\{\alpha_{n+1}^{h}=j\right\}=q_{i j} \tilde{\Delta} t^{h}(x, i, u)+o\left(\tilde{\Delta} t^{h}(x, i, u)\right), \\
& \text { for } j \neq i \text {, } \\
& P_{x, i, n}^{u, h, 2}\left\{\alpha_{n+1}^{h}=i\right\}=1+q_{i i} \tilde{\Delta} t^{h}(x, i, u)+o\left(\tilde{\Delta} t^{h}(x, i, u)\right), \\
& \sup _{n, \omega \in \Omega}\left|\Delta \xi_{n}^{h}\right| \rightarrow 0 \text { as } h \rightarrow 0 .
\end{aligned}
$$

The impulse and singular controls can be seen as a combination of capital injection $\left(I_{n}^{h}=0\right)$ and dividend payment part $\left(I_{n}^{h}=1\right)$. Also we require the impulse and singular controls to be "impulsive" or "instantaneous." Let $\widetilde{i}$ In other words, the interpolation interval on $S_{h} \times$
$\mathcal{M} \times U \times\{0,1,2\}$ is

$$
\begin{aligned}
& \Delta t^{h}(x, i, u, \widetilde{i})=\tilde{\Delta} t^{h}(x, i, u) I_{\{\widetilde{i}=2\}}, \\
& \text { for any }(x, i, u, \widetilde{i}) \in S_{h} \times \mathcal{M} \times U \times\{0,1,2\} .
\end{aligned}
$$

Denote by $\pi^{h}:=\left\{\pi_{n}^{h}, n \geq 0\right\}$ the sequence of control actions, where

$$
\pi_{n}^{h}:=\Delta l_{n}^{h} I_{\left\{I_{n}^{h}=0\right\}}+\Delta z_{n}^{h} I_{\left\{I_{n}^{h}=1\right\}}+u_{n}^{h} I_{\left\{I_{n}^{h}=2\right\}} .
$$

The sequence $\pi^{h}$ is said to be admissible if $\pi_{n}^{h}$ is $\sigma\left\{\left(\xi_{0}^{h}, \alpha_{0}^{h}\right), \ldots,\left(\xi_{n}^{h}, \alpha_{n}^{h}\right), \pi_{0}^{h}, \ldots, \pi_{n-1}^{h}\right\}$-adapted and for any $E \in \mathcal{B}\left(S_{h} \times \mathcal{M}\right)$, we have

$$
\begin{array}{r}
P\left\{\left(\xi_{n+1}^{h}, \alpha_{n+1}^{h}\right) \in E \mid \sigma\left\{\left(\xi_{0}^{h}, \alpha_{0}^{h}\right), \ldots,\left(\xi_{n}^{h}, \alpha_{n}^{h}\right),\right.\right. \\
\left.\left.\pi_{0}^{h}, \ldots, \pi_{n}^{h}\right\}\right\}=p^{h}\left(\left(\xi_{n}^{h}, \alpha_{n}^{h}\right), E \mid \pi_{n}^{h}\right),
\end{array}
$$

and

$$
\begin{aligned}
& P\left\{\left(\xi_{n+1}^{h}, \alpha_{n+1}^{h}\right)=(B, i) \mid\left(\xi_{n}^{h}, \alpha_{n}^{h}\right)\right. \\
& \left.\quad=(B+h, i), \sigma\left\{\left(\xi_{0}^{h}, \alpha_{0}^{h}\right), \ldots,\left(\xi_{n}^{h}, \alpha_{n}^{h}\right), \pi_{0}^{h}, \ldots, \pi_{n}^{h}\right\}\right\}=1 .
\end{aligned}
$$

Let

$$
\begin{aligned}
& t_{0}^{h}:=0, t_{n}^{h}:=\sum_{k=0}^{n-1} \Delta t^{h}\left(\xi_{k}^{h}, \alpha_{k}^{h}, u_{k}^{h}, I_{k}^{h}\right), \\
& n^{h}(t):=\max \left\{n: t_{n}^{h} \leq t\right\} .
\end{aligned}
$$

Then the piecewise constant interpolations, denoted by $\left(\xi^{h}(\cdot), \alpha^{h}(\cdot)\right), u^{h}(\cdot), l^{h}(\cdot)$, and $z^{h}(\cdot)$, are naturally defined as

$$
\begin{aligned}
& \xi^{h}(t)=\xi_{n}^{h}, \alpha^{h}(t)=\alpha_{n}^{h}, u^{h}(t)=u_{n}^{h}, \\
& l^{h}(t)=\sum_{k \leq n^{h}(t)} \Delta l_{k}^{h} I_{\left\{I_{k}^{h}=2\right\}}, z^{h}(t)=\sum_{k \leq n^{h}(t)} \Delta z_{k}^{h} I_{\left\{I_{k}^{h}=0\right\}},
\end{aligned}
$$

for $t \in\left[t_{n}^{h}, t_{n+1}^{h}\right)$. Let $\eta_{h}:=\inf \left\{n: \xi_{n}^{h} \in \partial G\right\}$. Then the first exit time of $\xi^{h}$ from $G$ is $\tau^{h}=t_{\eta_{h}}^{h}$. Let $\left(\xi_{0}^{h}, \alpha_{0}^{h}\right)=$ $(x, i) \in S_{h} \times \mathcal{M}$ and $\pi^{h}$ be an admissible control. The cost function for the controlled Markov chain is defined as

$$
J_{B}^{h}\left(x, i, \pi^{h}\right)=E \sum_{k=1}^{\eta_{h}-1} e^{-r t_{k}^{h}} \Delta z_{k}^{h},
$$

which is analogous to (2.4) regarding to the definition of interpolation intervals in (3.6). The value function of the controlled Markov chain is

$$
V_{B}^{h}(x, i)=\sup _{\pi^{h} \text { admissible }} J_{B}^{h}\left(x, i, \pi^{h}\right) .
$$

We shall show that $V_{B}^{h}(x, i)$ satisfies the dynamic pro- 


$$
\begin{aligned}
& V_{B}^{h}(x, i) \\
& =\left\{\begin{array}{l}
\max _{u \in U}\left\{\sum_{(y, j)} e^{-r \Delta t^{h}(x, i, u, 2)} p^{h}((x, i),(y, j) \mid \pi) V^{h}(y, j),\right. \\
\sum_{(y, j)} p^{h}((x, i),(y, j) \mid \pi) V^{h}(y, j)+\beta_{1} h, \\
\left.\sup _{0 \leq \widetilde{y} \leq B-x} V^{h}(x+\widetilde{y}, i)-\beta_{2} \widetilde{y}-K\right\}, \text { for } x \in S_{h}, \\
0, \quad \text { for } x=0 .
\end{array}\right.
\end{aligned}
$$

Note that discount does not appear in the second and third line above because the singular and impulse control are instantaneous. In the actual computing, we use iteration in value space or iteration in policy space together with Gauss-Seidel iteration to solve $V^{h}$. The computations will be very involved. In contrast to the usual state space $S_{h}$ in [Kushner and Dupuis, 2001], here we need to deal with an enlarged state space $S_{h} \times \mathcal{M}$ due to the presence of regime switching.

\subsection{Discretization}

Define the approximation to the first and the second derivatives of $V(\cdot, i)$ by finite difference method in the first part of QVIs (2.11) using stepsize $h>0$ as:

$$
\begin{aligned}
& V(x, i) \rightarrow V^{h}(x, i) \\
& V_{x}(x, i) \rightarrow \frac{V^{h}(x+h, i)-V^{h}(x, i)}{h} \\
& \quad \text { for }[g(i)(1-u)+u b(i)] x+c(i)>0, \\
& V_{x}(x, i) \rightarrow \frac{V^{h}(x, i)-V^{h}(x-h, i)}{h} \\
& \quad \text { for }[g(i)(1-u)+u b(i)] x+c(i)<0, \\
& V_{x x}(x, i) \rightarrow \frac{V^{h}(x+h, i)-2 V^{h}(x, i)+V^{h}(x-h, i)}{h^{2}} .
\end{aligned}
$$

For the second part of the QVIs, we choose

$$
V_{x}(x, i) \rightarrow \frac{V^{h}(x, i)-V^{h}(x-h, i)}{h} .
$$

$$
\begin{aligned}
& \max _{u \in U}\left\{\frac{V^{h}(x+h, i)-V^{h}(x, i)}{h}[[g(i)(1-u)+u b(i)] x\right. \\
& +c(i)]^{+}-\frac{V^{h}(x, i)-V^{h}(x-h, i)}{h}[[g(i)(1-u)+u b(i)] \\
& \times x+c(i)]^{-}+\frac{V^{h}(x+h, i)-2 V^{h}(x, i)+V^{h}(x-h, i)}{h^{2}} \\
& \times \frac{u^{2} \sigma^{2}(i) x^{2}}{2}+\sum_{j} V^{h}(x, \cdot) q_{i j}-r V^{h}(x, i), \\
& \beta_{1}-\frac{V^{h}(x, i)-V^{h}(x-h, i)}{h}, \\
& \left.\quad \sup _{0 \leq \widetilde{y} \leq B-x} V^{h}(x+\widetilde{y}, i)-\beta_{2} \widetilde{y}-K\right\}=0, \\
& \quad \forall x \in S_{h}, i \in \mathcal{M} \text { with the boundary condition } \\
& \quad V^{h}(x, i)=0, \text { for } x=0,
\end{aligned}
$$

where $[[g(i)(1-u)+u b(i)] x+c(i)]^{+}$and $[[g(i)(1-u)+$ $u b(i)] x+c(i)]^{-}$are the positive and negative parts of $[[g(i)(1-u)+u b(i)] x+c(i)]$, respectively.

We set the effective average discount factor as

$$
\begin{aligned}
& \frac{1}{1+r \Delta t^{h}(x, i, u, 2)} \\
& =\exp \left[-r \Delta t^{h}(x, i, u, 2)\left(1+O\left(\Delta t^{h}(x, i, u, 2)\right)\right)\right]
\end{aligned}
$$

Then (3.10) can be rewritten as

$$
\begin{aligned}
& V_{B}^{h}(x, i) \\
& =\left\{\begin{array}{l}
\max _{u \in U}\left\{\sum_{(y, j)} \frac{1}{1+r \Delta t^{h}(x, i, u, 2)} p^{h}((x, i),(y, j) \mid \pi)\right. \\
\times V^{h}(y, j), \sum_{(y, j)} p^{h}((x, i),(y, j) \mid \pi) V^{h}(y, j)+\beta_{1} h, \\
\left.\sup _{0 \leq \widetilde{y} \leq B-x} V^{h}(x+\widetilde{y}, i)-\beta_{2} \widetilde{y}-K\right\}, \text { for } x \in S_{h}, \\
0, \text { for } x=0 .
\end{array}\right.
\end{aligned}
$$

Simplifying (3.12) and comparing with (3.13), we achieve the transition probabilities of the first part of the right 
side of (3.10) as the following:

$$
\begin{aligned}
& p_{D}^{h}((x, i),(x+h, i) \mid \pi) \\
& =\frac{\sigma^{2}(i) u^{2} x^{2} / 2+h[[g(i)(1-u)+u b(i)] x+c(i)]^{+}}{D-r h^{2}}, \\
& p_{D}^{h}((x, i),(x-h, i) \mid \pi) \\
& =\frac{\sigma^{2}(i) u^{2} x^{2} / 2+h[[g(i)(1-u)+u b(i)] x+c(i)]^{-}}{D-r h^{2}}, \\
& p_{D}^{h}((x, i),(x, j) \mid \pi)=\frac{h^{2}}{D-r h^{2}} q_{i j}, \text { for } i \neq j, \\
& p_{D}^{h}(\cdot)=0, \text { otherwise, } \\
& \Delta t^{h}(x, i, u, 2)=\frac{h^{2}}{D},
\end{aligned}
$$

with

$D=\sigma^{2}(i) u^{2} x^{2}+h|[g(i)(1-u)+u b(i)] x+c(i)|+h^{2}\left(r-q_{i i}\right)$

being well defined. We also find the transition probability for the second part of the right hand side of (3.10). That is,

$$
p_{D}^{h}((x, i),(x-h, i) \mid \pi)=1 .
$$

Suppose that the current state is $\xi_{n}^{h}=x, \alpha_{n}^{h}=i$, and the control is $u_{n}^{h}=u$. Next interpolation interval $\Delta t^{h}(x, i, u)$ is determined by (3.14). To present the claim terms, we determine the next state $\left(\xi_{n+1}^{h}, \alpha_{n+1}^{h}\right)$ by noting:

1. No claims occur in $\left[t_{n}^{h}, t_{n+1}^{h}\right)$ with probability $\left(1-\lambda \Delta t^{h}(x, i, u, 2)+o\left(\Delta t^{h}(x, i, u, 2)\right)\right)$; we determine $\left(\xi_{n+1}^{h}, \alpha_{n+1}^{h}\right)$ by transition probability $p_{D}^{h}(\cdot)$ as in $(3.14)$.

2. There is a claim in $\left[t_{n}^{h}, t_{n+1}^{h}\right)$ with probability $\left.\lambda \Delta t^{h}(x, i, u)+o\left(\Delta t^{h}(x, i, u, 2)\right)\right)$, we determine $\left(\xi_{n+1}^{h}, \alpha_{n+1}^{h}\right)$ by

$$
\xi_{n+1}^{h}=\xi_{n}^{h}-q_{h}(x, i, \rho), \alpha_{n+1}^{h}=\alpha_{n}^{h},
$$

where $\rho \sim \Pi(\cdot)$, and $q_{h}(x, i, \rho) \in S_{h} \subseteq \Gamma$ such that $q_{h}(x, i, \rho)$ is the nearest value of $q(x, i, \rho)$ so that $\xi_{n+1}^{h} \in S_{h}$. Then $\left|q_{h}(x, i, \rho)-q(x, i, \rho)\right| \rightarrow 0$ as $h \rightarrow 0$, uniformly in $x$.

Let $H_{n}^{h}$ denote the event that $\left(\xi_{n+1}^{h}, \alpha_{n+1}^{h}\right)$ is determined by the first alternative above and use $T_{n}^{h}$ to denote the event of the second case. Let $I_{H_{n}^{h}}$ and $I_{T_{n}^{h}}$ be corresponding indicator functions, respectively. Then $I_{H_{n}^{h}}+I_{T_{n}^{h}}=$ 1. Then we need a new definition of the local consistency for Markov chain approximation of compound Poisson process with diffusion and regime-switching.

Definition 2 A controlled Markov chain $\left\{\left(\xi_{n}^{h}, \alpha_{n}^{h}\right), n<\right.$ $\infty\}$ is said to be locally consistent with $(2.3)$, if there is an interpolation interval $\Delta t^{h}(x, i, u, 2) \rightarrow 0$ as $h \rightarrow 0$ uniformly in $x, i$, and $u$ such that
1. there is a transition probability $p_{D}^{h}(\cdot)$ that is locally consistent with (3.3) in the sense that (3.5) holds.

2. there is a $\delta^{h}(x, i, u, 2)=o\left(\Delta t^{h}(x, i, u, 2)\right)$ such that the one-step transition probability $\left\{p^{h}((x, i)\right.$, $(y, j)) \mid \pi\}$ is given by

$$
\begin{aligned}
& p^{h}(((x, i),(y, j)) \mid \pi)=\left(1-\lambda \Delta t^{h}(x, i, u, 2)\right. \\
& \left.\quad+\delta^{h}(x, i, u, 2)\right) p_{D}^{h}((x, i),(y, j)) \\
& \quad+\left(\lambda \Delta t^{h}(x, i, u, 2)+\delta^{h}(x, i, u, 2)\right) \\
& \quad \times \Pi\left\{\rho: q_{h}(x, i, \rho)=x-y\right\} .
\end{aligned}
$$

Furthermore, the system of dynamic programming equations is a modification of (3.10). That is,

$$
\begin{aligned}
& V^{h}(x, i) \\
& = \begin{cases}\max _{\pi \in \mathcal{A}}\left[\left(1-\lambda \Delta t^{h}(x, i, u, 2)+\delta^{h}(x, i, u, 2)\right)\right. \\
\times e^{-r \Delta t^{h}(x, i, u, 2)} \sum_{(y, j)}\left(p_{D}^{h}((x, i),(y, j)) \mid \pi\right) V^{h}(y, i) \\
+\left(\lambda \Delta t^{h}(x, i, u, 2)+\delta^{h}(x, i, u, 2)\right) e^{-r \Delta t^{h}(x, i, u, 2)} \\
\times \int_{0}^{\infty} V^{h}\left(x-q_{h}(x, i, \rho), i\right) \Pi(d \rho), \\
V^{h}(x-h, i)+\beta_{1} h, \\
\left.\sup _{0 \leq \tilde{y} \leq B-x} V^{h}(x+\widetilde{y}, i)-\beta_{2} \widetilde{y}-K\right], & \text { for } x \in S_{h}, \\
\sup _{0 \leq \tilde{y} \leq B} V^{h}(\widetilde{y}, i)-\beta_{2} \widetilde{y}-K, & \text { for } x=0, \\
V^{h}(B-h, i)+\beta_{1} h, & \text { for } x=B .\end{cases}
\end{aligned}
$$

Remark 3 The first part of the QVIs can be seen as a "continuation" region where the regular control of investment is dominant. The Markov approximating chain can switch between regimes and states nearby with the transition probabilities defined above. The second part of the QVIs refers to the "dividend payment" region, where the dividends are paid out and the singular control is dominant. Due to the representation of singular control, the Markov chain will be reflected back one step $h$ w.p. 1 on the boundary. The third part of the QVIs is the "capital injection" region, where extra capitals will be ordered and injected immediately when surplus is sufficiently low and hits the free boundary of "capital injection" region.

In real world, the wealth process can not be arbitrarily high. Thus, we need define an upper boundary of wealth for practical computation. Our ultimate goal is to show $V^{h}$ converges to $V$ in a large enough interval $[0, B]$ as $h \rightarrow 0$. As in [Kushner and Dupuis, 2001], in the verification of the convergence of approximation sequence, we need to show that the approximating sequence is tight and then appropriately characterize the subsequential weak limit, which does not hold in the 
case of unrestrict dividend payment process. To overcome this difficulty, we adapt the techniques developed in [Kushner and Martins, 1991]. The basic idea is to suitably re-scale the time so that the processes involved in the convergence analysis are tight in the new time scale; carry out weak convergence analysis with the rescaled processes; and revert back to the original time scale to obtain the convergence of approximating sequence to the original value function.

\section{Convergence of Numerical Approximation}

This section focuses on the asymptotic properties of the approximating Markov chain proposed in the last section. The main techniques are methods of weak convergence. To begin with, the technique of time rescaling and the interpolation of the approximation sequences is introduced in Section 4.1. The definition of relax controls is presented in Section 4.2. Section 4.3 deals with weak convergence of $\left\{\widehat{\xi}^{h}(\cdot), \widehat{\alpha}^{h}(\cdot), \widehat{m}^{h}(\cdot), \widehat{W}^{h}(\cdot), \widehat{N}(\cdot), \widehat{R}^{h}(\cdot), \widehat{z}^{h}(\cdot), \widehat{l}^{h}(\cdot), \widehat{T}^{h}(\cdot)\right\}$ a sequence of rescaled process. As a result, a sequence of controlled surplus processes converges to a limit surplus process. By using the techniques of inversion, Section 4.3 also takes up the issue of the weak convergence of the surplus process. The chattering lemmas of optimal control is presented in 4.4. Finally Section 4.5 establishes the convergence of the value function.

\subsection{Interpolation and Rescaling}

Based on the approximating Markov chain constructed above, the piecewise constant interpolation is obtained and the appropriate interpolation interval level is chosen. Recalling (3.7), the continuous-time interpolations $\left(\xi^{h}(\cdot), \alpha^{h}(\cdot)\right), u^{h}(\cdot), z^{h}(\cdot)$, and $l^{h}(\cdot)$ are defined. In addition, let $\mathcal{U}^{h}$ denote the collection of controls, which are determined by a sequence of measurable functions $F_{n}^{h}(\cdot)$ such that

$$
u_{n}^{h}=F_{n}^{h}\left(\xi_{k}^{h}, \alpha_{k}^{h}, k \leq n ; u_{k}^{h}, k \leq n\right) .
$$

Let the discrete times at which claims occur be denoted by $\nu_{j}^{h}, j=1,2, \ldots$ Then we have

$$
\xi_{\nu_{j-1}^{h}}^{h}-\xi_{\nu_{j}^{h}}^{h}=q_{h}\left(\xi_{\nu_{j-1}^{h}}^{h}, \alpha_{\nu_{j-1}^{h}}^{h}, \rho\right)
$$

Define $\mathcal{D}_{n}^{h}$ as the smallest $\sigma$-algebra of $\left\{\xi_{k}^{h}, \alpha_{k}^{h}, u_{k}^{h}, H_{k}^{h}\right.$, $\left.z_{k}^{h}, l_{k}^{h}, k \leq n ; \nu_{k}^{h}, \rho_{k}^{h}: \nu_{k}^{h} \leq t_{n}\right\}$. In addition, $\mathcal{U}^{h}$ defined by (4.1) is equivalent to the collection of all piecewise constant admissible controls with respect to $\mathcal{D}_{n}^{h}$.

Using the representations of regular control, singular control, impulse control and the interpolations defined above, (3.4) yields

$$
\begin{aligned}
\xi_{n}= & x+\sum_{k=0}^{n-1}\left[\Delta \xi_{k}^{h} I_{H_{k}^{h}}+\Delta \xi_{k}^{h}\left(1-I_{H_{k}^{h}}\right)\right]-\sum_{k=0}^{n-1} z_{k}^{h}-\sum_{k=0}^{n-1} l_{k}^{h} \\
= & x+\sum_{k=0}^{n-1} E_{k}^{h} \Delta \xi_{k}^{h} I_{H_{k}^{h}}+\sum_{k=0}^{n-1}\left(\Delta \xi_{k}^{h}-E_{k}^{h} \Delta \xi_{k}^{h}\right) I_{H_{k}^{h}} \\
& +\sum_{k=0}^{n-1} \Delta \xi_{k}^{h}\left(1-I_{H_{k}^{h}}\right)-\sum_{k=0}^{n-1} z_{k}^{h}-\sum_{k=0}^{n-1} l_{k}^{h} .
\end{aligned}
$$

The local consistency leads to

$$
\begin{aligned}
& \sum_{k=0}^{n-1} E_{k}^{h} \Delta \xi_{k}^{h} I_{H_{k}^{h}}=\sum_{k=0}^{n-1}\left[\left[g\left(\alpha_{k}^{h}\right)\left(1-u_{k}^{h}\right)+u_{k}^{h} b\left(\alpha_{k}^{h}\right)\right] \xi_{k}^{h}\right. \\
& \left.\quad+c\left(\alpha_{k}^{h}\right)\right] \Delta t_{k}^{h}+o\left(\Delta t_{k}^{h}\right) I_{H_{k}^{h}} \\
& =\sum_{k=0}^{n-1}\left[\left[g\left(\alpha_{k}^{h}\right)\left(1-u_{k}^{h}\right)+u_{k}^{h} b\left(\alpha_{k}^{h}\right)\right] \xi_{k}^{h}+c\left(\alpha_{k}^{h}\right)\right] \Delta t_{k}^{h} \\
& \quad+o\left(\Delta t_{k}^{h}\right)-\left(\max _{k^{\prime} \leq n} \Delta t_{k^{\prime}}^{h}\right) O\left(\sum_{k=0}^{n-1} I_{T_{k}^{h}}\right) .
\end{aligned}
$$

Denote

$$
\begin{aligned}
& M_{n}^{h}=\sum_{k=0}^{n-1}\left(\Delta \xi_{k}^{h}-E_{k}^{h} \Delta \xi_{k}^{h}\right) I_{H_{k}^{h}}, \\
& R_{n}^{h}=-\sum_{k=0}^{n-1} \Delta \xi_{k}^{h}\left(1-I_{H_{k}^{h}}\right)=\sum_{k: \nu_{k}<n} q_{h}\left(\xi_{\nu_{k}}^{h}, \alpha_{\nu_{k}}^{h}, \rho_{k}\right),
\end{aligned}
$$

where $M_{n}^{h}$ is a martingale with respect to $\mathcal{D}_{n}^{h}$. Note that

$E \sum_{k=0}^{n-1} I_{T_{k}^{h}}=E\left[\right.$ number of $\left.n: \nu_{n}^{h} \leq t\right] \rightarrow \lambda t \quad$ as $\quad h \rightarrow 0$.

This implies

$$
\left(\underset{\widetilde{k} \leq n}{\max } \Delta t_{k}^{h}\right) O\left(\sum_{k=0}^{n-1} I_{T_{k}^{h}}\right) \rightarrow 0 \text { in probability as } h \rightarrow 0 .
$$

Hence we can drop the term involving $I_{T_{k}^{h}}$ without affecting the limit in (4.3). We attempt to represent $M^{h}(t)$ similar to the diffusion term in (2.3). Define $W^{h}(\cdot)$ as

$$
\begin{aligned}
W^{h}(t) & =\sum_{k=0}^{n-1}\left(\Delta \xi_{k}^{h}-E_{k}^{h} \Delta \xi_{k}^{h}\right) /\left[\sigma\left(\alpha^{h}(s)\right) u^{h}(s) \xi^{h}(s)\right] \\
& =\int_{0}^{t}\left[\sigma\left(\alpha^{h}(s)\right) u^{h}(s) \xi^{h}(s)\right]^{-1} d M^{h}(s)
\end{aligned}
$$




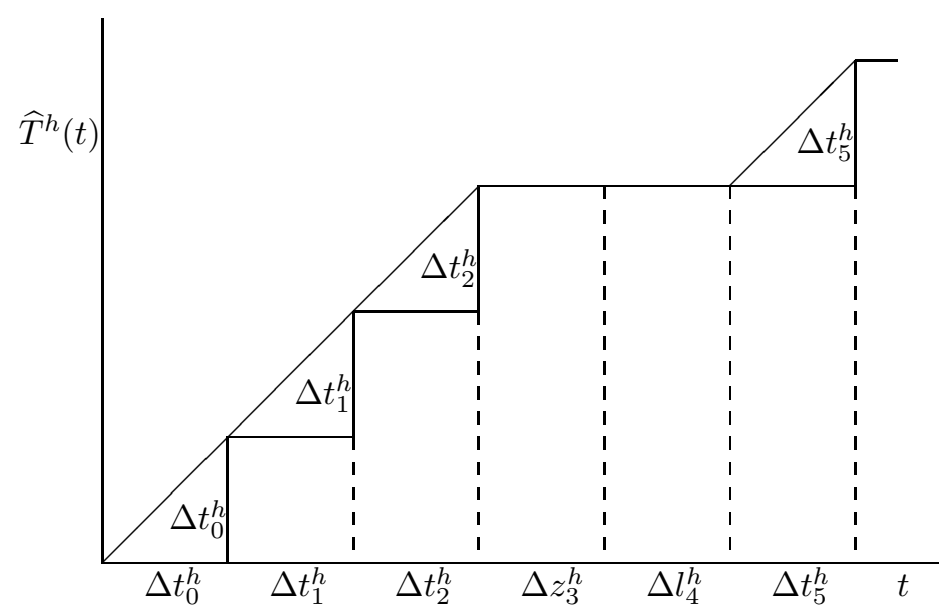

Fig. 4.1. The stretched out time scale

Combining (4.3)-(4.5), we rewrite (4.2) by

$$
\begin{aligned}
& \xi^{h}(t)=x+\int_{0}^{t}\left[\left[g\left(\alpha^{h}(s)\right)\left(1-u^{h}(s)\right)+u^{h}(s) b\left(\alpha^{h}(s)\right)\right]\right. \\
& \left.\times \xi^{h}(s)+c\left(\alpha^{h}(s)\right)\right] d s+\int_{0}^{t} \sigma\left(\alpha^{h}(s)\right) u^{h}(s) \xi^{h}(s) d W^{h}(s) \\
& -R^{h}(t)-z^{h}(t)-l^{h}(t)+\varepsilon^{h}(t),
\end{aligned}
$$

where $R^{h}(t)=\sum_{\nu_{n}^{h} \leq t} q_{h}\left(\xi_{\nu_{n}^{-}}^{h}, \alpha_{\nu_{n}}^{h}, \rho_{n}\right)$, and $\varepsilon^{h}(t)$ is a negligible error satisfying

$$
\lim _{h \rightarrow \infty} \sup _{0 \leq t \leq T} E\left|\varepsilon^{h}(t)\right| \rightarrow 0 \text { for any } 0<T<\infty .
$$

Next we introduce the rescaling process. The basic idea of rescaling time is to "stretch out" the control and state processes so that they are "smoother" so the tightness of $l^{h}(\cdot)$ and $z^{h}(\cdot)$ can be proved. Define $\Delta \widehat{t}_{n}^{h}$ by

$\Delta \widehat{t}_{n}^{h}= \begin{cases}\Delta t_{n}^{h} \quad \text { for a diffusion on step } n \\ \left|\Delta z_{n}^{h}\right|=h \quad \text { for a dividend payment on step } n \\ \left|\Delta g_{n}^{h}\right|=h \quad \text { for a capital injection on step } n\end{cases}$

and define $\widehat{T}^{h}(\cdot)$ by $\widehat{T}^{h}(t)=\sum_{i=0}^{n-1} \Delta t_{i}^{h}=t_{n}^{h}$ for $t \in$ $\left[\widehat{t}_{n}^{h}, \widehat{t}_{n+1}^{h}\right]$. Thus, $\widehat{T}^{h}(\cdot)$ will increase with the slope of unity if and only if a regular control is exerted.

In addition, define the rescaled and interpolated process $\widehat{\xi}^{h}(t)=\xi^{h}\left(\widehat{T}^{h}(t)\right)$, and define $\widehat{\alpha}^{h}(t), \widehat{u}^{h}(t), \widehat{N}^{h}(\cdot), \widehat{R}^{h}(t)$, $\widehat{z}^{h}(t)$ and $\widehat{l}^{h}(t)$ similarly. The time scale is stretched out by $h$ at the impulse and singular control steps. We can now write

$$
\begin{aligned}
& \widehat{\xi}^{h}(t)=x+\int_{0}^{t}\left[\left[g\left(\widehat{\alpha}^{h}(s)\right)\left(1-\widehat{u}^{h}(s)\right)+\widehat{u}^{h}(s) b\left(\widehat{\alpha}^{h}(s)\right)\right]\right. \\
& \left.\times \widehat{\xi}^{h}(s)+c\left(\widehat{\alpha}^{h}(s)\right)\right] d s+\int_{0}^{t} \sigma\left(\widehat{\alpha}^{h}(s)\right) \widehat{u}^{h}(s) \widehat{\xi}^{h}(s) d W^{h}(s) \\
& -\widehat{R}^{h}(t)-\widehat{z}^{h}(t)-\widehat{l}^{h}(t)+\varepsilon^{h}(t) .
\end{aligned}
$$

\subsection{Relaxed Controls}

Let $\mathcal{B}(U \times[0, \infty))$ be the $\sigma$-algebra of Borel subsets of $U \times$ $[0, \infty)$. An admissible relaxed control (or deterministic relaxed control $) m(\cdot)$ is a measure on $\mathcal{B}(U \times[0, \infty))$ such that $m(U \times[0, t])=t$ for each $t \geq 0$. Given a relaxed control $m(\cdot)$, there is an $m_{t}(\cdot)$ such that $m(d \psi d t)=$ $m_{t}(d \psi) d t$. We can define $m_{t}(B)=\lim _{\delta \rightarrow 0} \frac{m(B \times[t-\delta, t])}{\delta}$ for $B \in \mathcal{B}(U)$. With the given probability space, we say that $m(\cdot)$ is an admissible relaxed (stochastic) control for $(W(\cdot), \alpha(\cdot))$ or $(m(\cdot), W(\cdot), \alpha(\cdot))$ is admissible, if $m(\cdot, \omega)$ is a deterministic relaxed control with probability one and if $m(A \times[0, t])$ is $\mathcal{F}_{t}$-adapted for all $A \in \mathcal{B}(U)$. There is a derivative $m_{t}(\cdot)$ such that $m_{t}(\cdot)$ is $\mathcal{F}_{t}$-adapted for all $A \in \mathcal{B}(U)$.

Given a relaxed control $m^{h}(\cdot)$ of $u^{h}(\cdot)$, we define the derivative $m_{t}(\cdot)$ such that

$$
m^{h}(K)=\int_{U \times[0, \infty)} I_{\left\{\left(u^{h}, t\right) \in K\right\}} m_{t}(d \psi) d t
$$

for all $K \in \mathcal{B}(U \times[0, \infty))$, and that for each $t, m_{t}(\cdot)$ is a measure on $\mathcal{B}(U)$ satisfying $m_{t}(U)=1$. For example, we can define $m_{t}(\cdot)$ in any convenient way for $t=0$ and as the left-hand derivative for $t>0$,

$$
m_{t}(A)=\lim _{\delta \rightarrow 0} \frac{m(A \times[t-\delta, t])}{\delta}, \forall A \in \mathcal{B}(U) .
$$

Note that $m(d \psi d t)=m_{t}(d \psi) d t$. It is natural to define the relaxed control representation $m^{h}(\cdot)$ of $u^{h}(\cdot)$ by

$$
m_{t}^{h}(A)=I_{\left\{u^{h}(t) \in A\right\}}, \forall A \in \mathcal{B}(U) .
$$

Let $\mathcal{F}_{t}^{h}$ be a filtration, which denotes the minimal $\sigma$ algebra that measures

$$
\begin{array}{r}
\left\{\xi^{h}(s), \alpha^{h}(\cdot), m_{s}^{h}(\cdot), W^{h}(s), N^{h}(s), R^{h}(s), z^{h}(s),\right. \\
\left.l^{h}(s), s \leq t\right\} .
\end{array}
$$

Use $\Gamma^{h}$ to denote the set of $\pi^{h}(\cdot)=\left(m^{h}(\cdot), z^{h}(\cdot), l^{h}(\cdot)\right)$, where $m^{h}(\cdot)$ is the admissible relaxed controls with respect to $\left(\alpha^{h}(\cdot), W^{h}(\cdot)\right)$ such that $m_{t}^{h}(\cdot)$ is a fixed probability measure in the interval $\left[t_{n}^{h}, t_{n+1}^{h}\right)$ given $\mathcal{F}_{t}^{h}$. Referring to the stretched out time scale, we denote the 
rescaled relax control as $m_{\widehat{T}^{h}(t)}(d \psi)$. Define $M_{t}(A)$ and $M_{t}^{h}(d \psi)$ by

$$
\begin{aligned}
& M_{t}(A) d t=d W(t) I_{u(t) \in A}, \forall A \in \mathcal{B}(U) \\
& M_{t}^{h}(d \psi) d t=d W^{h}(t) I_{u^{h}(t) \in \mathcal{U}} .
\end{aligned}
$$

Analogously, as an extension of time rescaling, we let

$$
\widehat{M}_{\widehat{T}^{h}(t)}^{h}(d \psi) d \widehat{T}^{h}(t)=d \widehat{W}^{h}\left(\widehat{T}^{h}(t)\right) I_{u^{h}\left(\widehat{T}^{h}(t)\right) \in \mathcal{U}} .
$$

With the notation of relaxed control given above, we can write (4.6), (4.9), and the value function (2.5) as

$$
\begin{gathered}
\xi^{h}(t)=x+\int_{0}^{t} \int_{\mathcal{U}}\left[g\left(\alpha^{h}(s)\right)(1-\psi)+\psi b\left(\alpha^{h}(s)\right)\right] \xi^{h}(s) \\
\left.+c\left(\alpha^{h}(s)\right)\right] m_{s}^{h}(d \psi) d s \\
+\int_{0}^{t} \int_{\mathcal{U}} \psi \sigma\left(\alpha^{h}(s)\right) \xi^{h}(s) M_{s}^{h}(d \psi) d s \\
-R^{h}(t)-z^{h}(t)-l^{h}(t)+\varepsilon^{h}(t), \\
\widehat{\xi}^{h}(t)=x+\int_{0}^{t} \int_{\mathcal{U}}\left[\left[g\left(\widehat{\alpha}^{h}(s)\right)(1-\psi)+\psi b\left(\widehat{\alpha}^{h}(s)\right)\right]\right. \\
\left.\quad \times \widehat{\xi}^{h}(s)+c\left(\widehat{\alpha}^{h}(s)\right)\right] \widehat{m}_{\widehat{T}^{h}(s)}(d \psi) d \widehat{T}^{h}(s) \\
\quad+\int_{0}^{t} \int_{\mathcal{U}} \psi \sigma\left(\widehat{\alpha}^{h}(s)\right) \widehat{\xi}^{h}(s) \widehat{M}_{\widehat{T}^{h}(s)}(d \psi) d \widehat{T}^{h}(s) \\
\quad-\widehat{R}^{h}(t)-\widehat{z}^{h}(t)-\widehat{l}^{h}(t)+\varepsilon^{h}(t),
\end{gathered}
$$

and

$$
V^{h}(x, i)=\inf _{\pi^{h} \in \Gamma^{h}} J^{h}\left(x, i, \pi^{h}\right) .
$$

Now we give the definition of existence and uniqueness of weak solution.

Definition 4 By a weak solution of (4.14), we mean that there exists a probability space $(\Omega, \mathcal{F}, P)$, a filtration $\mathcal{F}_{t}$, and process $(X(\cdot), \alpha(\cdot), m(\cdot), W(\cdot), N(\cdot))$ such that $W(\cdot)$ is a standard $\mathcal{F}_{t}$-Wiener process, $\alpha(\cdot)$ is a Markov chain with generator $Q$ and state space $\mathcal{M}$, for each $i \in \mathcal{M}, N(\cdot)$ is an $\mathcal{F}_{t}$-Poisson process, $m(\cdot)$ is admissible with respect to $X(\cdot)$, and is $\mathcal{F}_{t^{-}}$ adapted, and (4.14) is satisfied. For an initial condition $(x, i)$, by the weak sense uniqueness, we mean that the probability law of the admissible process $(\alpha(\cdot), m(\cdot), W(\cdot), N(\cdot))$ determines the probability law of solution $(X(\cdot), \alpha(\cdot), m(\cdot), W(\cdot), N(\cdot))$ to (4.14), irrespective of probability space.

To proceed, we need the following assumptions.

(A) Let $u(\cdot)$ be an admissible ordinary control with respect to $W(\cdot), \alpha(\cdot)$, and $N(\cdot)$, and suppose that $u(\cdot)$ is piecewise constant and takes only a finite number of values. For each initial condition, there exists a solution to $(4.14)$, where $m(\cdot)$ is the relaxed control representation of $u(\cdot)$ and this solution is unique in the weak sense.

\subsection{Convergence of A Sequence of Surplus Processes}

Lemma 5 Assume (A). Using the transition probabilities $\left\{p^{h}(\cdot)\right\}$ defined in (3.14), the interpolated process of the constructed Markov chain $\left\{\widehat{\alpha}^{h}(\cdot)\right\}$ converges weakly to $\widehat{\alpha}(\cdot)$, the rescaled Markov chain with generator $Q=$ $\left(q_{i j}\right)$.

Proof. Similar to Theorem 3.1 in [Yin et al., 2003], we can show that

$$
\begin{aligned}
& \left|E\left[\left(\alpha^{h}(t+s)-\alpha^{h}(t)\right)^{2}\right]\right| \mathcal{F}_{t}^{h} \mid \leq \bar{\gamma}^{h}(s) \\
& \text { and } \lim _{s \rightarrow 0} \limsup _{h \rightarrow 0} E \bar{\gamma}^{h}(s)=0,
\end{aligned}
$$

where $\bar{\gamma}^{h}(s) \geq 0$ is $\mathcal{F}_{t}^{h}$-measurable. On the other hand, due to the definition of $\widehat{\alpha}^{h}(\cdot)$, we have

$$
\begin{aligned}
& \left|E\left[\left(\widehat{\alpha}^{h}(t+s)-\widehat{\alpha}^{h}(t)\right)^{2}\right]\right| \mathcal{F}_{t}^{h} \mid \\
& \leq\left|E\left[\left(\alpha^{h}(t+s)-\alpha^{h}(t)\right)^{2}\right]\right| \mathcal{F}_{t}^{h} \mid \leq \bar{\gamma}^{h}(s) .
\end{aligned}
$$

Combining (4.17) and (4.18), we obtain $\widehat{\alpha}^{h}(\cdot)$ is tight. Furthermore, it can be shown that the constructed Markov chain $\left\{\widehat{\alpha}^{h}(\cdot)\right\}$ converges weakly to $\widehat{\alpha}(\cdot)$.

Theorem 6 Under the conditions of Lemma 5, let the approximating chain $\left\{\xi_{n}^{h}, \alpha_{n}^{h}, n<\infty\right\}$ be constructed with transition probabilities defined in (3.14) be locally consistent with $(2.3), \mathrm{m}^{h}(\cdot)$ be the relaxed control representation of $\left\{u_{n}^{h}, n<\infty\right\},\left(\xi^{h}(\cdot), \alpha^{h}(\cdot)\right)$ be the continuous-time interpolation defined in (3.7), and $\left\{\widehat{\xi}^{h}(\cdot), \widehat{\alpha}^{h}(\cdot), \widehat{m}^{h}(\cdot), \widehat{W}^{h}(\cdot), \widehat{N}^{h}(\cdot), \widehat{R}^{h}(\cdot), \widehat{z}^{h}(\cdot), \widehat{l}^{h}(\cdot)\right.$, $\left.\widehat{T}^{h}(\cdot)\right\}$ be the corresponding rescaled processes. Then $\left\{\widehat{\xi}^{h}(\cdot), \widehat{\alpha}^{h}(\cdot), \widehat{m}^{h}(\cdot), \widehat{W}^{h}(\cdot), \widehat{N}^{h}(\cdot), \widehat{R}^{h}(\cdot), \widehat{z}^{h}(\cdot), \widehat{l}^{h}(\cdot)\right.$, $\left.\widehat{T}^{h}(\cdot)\right\}$ is tight.

Proof. In view of Lemma $5,\left\{\widehat{\alpha}^{h}(\cdot)\right\}$ is tight. The sequence $\left\{\widehat{m}^{h}(\cdot)\right\}$ is tight since its range space is compact. Let $T<\infty$, and let $\tau_{h}$ be an $\mathcal{F}_{t}$-stopping time which is no bigger than $T$. Then for $\delta>0$,

$$
E_{\tau_{h}}^{u^{h}}\left(W^{h}\left(\tau_{h}+\delta\right)-W^{h}\left(\tau_{h}\right)\right)^{2}=\delta+\varepsilon_{h},
$$

where $\varepsilon_{h} \rightarrow 0$ uniformly in $\tau_{h}$. Taking limsup $\sup _{h \rightarrow 0}$ followed by $\lim _{\delta \rightarrow 0}$ yield the tightness of $\left\{W^{h}(\cdot)\right\}$. In view of [Kushner and Dupuis, 2001, Theorem 9.2.1], for each $i \in \mathcal{M}$, the sequence $\left\{N^{h}(\cdot)\right\}$ is tight because the mean number of claims on any bounded interval $[t, t+s]$ is 
bounded by $\lambda s+\delta_{1}^{h}(s)$, where $\delta_{1}^{h}(s)$ goes to zero as $h \rightarrow 0$, and

$$
\lim _{\delta \rightarrow 0} \inf _{h, n} P\left\{\nu_{n+1}^{h}-\nu_{n}^{h}>\delta \mid \text { data up to } \nu_{n}^{h}\right\}=1 .
$$

This also implies the tightness of $\left\{R^{h}(\cdot)\right\}$. Similar to the argument of $\widehat{\alpha}^{h}(\cdot)$, the tightness of $\left\{\widehat{N}^{h}(\cdot)\right\},\left\{\widehat{R}^{h}(\cdot)\right\}$ and $\widehat{W}^{h}(\cdot)$ is obtained. Furthermore, following the definition of "stretched out" timescale,

$$
\begin{aligned}
& \left|\widehat{z}^{h}\left(\tau_{h}+\delta\right)-\widehat{z}^{h}\left(\tau_{h}\right)\right| \leq|\delta|+O(h), \\
& \left|\widehat{l}^{h}\left(\tau_{h}+\delta\right)-\widehat{l}^{h}\left(\tau_{h}\right)\right| \leq|\delta|+O(h) .
\end{aligned}
$$

Thus $\left\{\widehat{z}^{h}(\cdot), \widehat{l}^{h}(\cdot)\right\}$ is tight. For notational simplicity, we assume that $b(\cdot)$ and $\sigma(\cdot)$ are bounded. For more general case, we can use a truncation device. These results and the boundedness of $b(\cdot)$ implies the tightness of $\left\{\xi^{h}(\cdot)\right\}$. Therefore it follows that $\left\{\widehat{\xi}^{h}(\cdot), \widehat{\alpha}^{h}(\cdot), \widehat{m}^{h}(\cdot), \widehat{W}^{h}(\cdot), \widehat{N}^{h}(\cdot), \widehat{R}^{h}(\cdot), \widehat{z}^{h}(\cdot), \widehat{l}^{h}(\cdot)\right.$, $\left.\widehat{T}^{h}(\cdot)\right\}$ is tight.

Since $\left\{\widehat{\xi}^{h}(\cdot), \widehat{\alpha}^{h}(\cdot), \widehat{m}^{h}(\cdot), \widehat{W}^{h}(\cdot), \widehat{N}^{h}(\cdot), \widehat{R}^{h}(\cdot), \widehat{z}^{h}(\cdot)\right.$, $\left.\widehat{l}^{h}(\cdot), \widehat{T}^{h}(\cdot)\right\}$ is tight, we can extract a weakly convergent subsequence, For simplicity, still index the subsequence by $h$. Denote the limit by $\{\widehat{X}(\cdot), \widehat{\alpha}(\cdot), \widehat{m}(\cdot), \widehat{W}(\cdot), \widehat{N}(\cdot)$, $\widehat{R}(\cdot), \widehat{z}(\cdot), \widehat{l}(\cdot), \widehat{T}(\cdot)\}$, whose paths are continuous w.p.1. We proceed to derive the following theorem, whose proof is provided in the appendix.

Theorem 7 Under the conditions of Theorem 6, let $\{\widehat{X}(\cdot), \widehat{\alpha}(\cdot), \widehat{m}(\cdot), \widehat{W}(\cdot), \widehat{N}(\cdot), \widehat{R}(\cdot), \widehat{z}(\cdot), \widehat{l}(\cdot), \widehat{T}(\cdot)\}$ be the limit of the weakly convergent subsequence of $\left\{\widehat{\xi}^{h}(\cdot), \widehat{\alpha}^{h}(\cdot), \widehat{m}^{h}(\cdot), \widehat{W}^{h}(\cdot), \widehat{N}^{h}(\cdot), \widehat{R}^{h}(\cdot), \widehat{z}^{h}(\cdot), \widehat{l}^{h}(\cdot)\right.$, $\left.\widehat{T}^{h}(\cdot)\right\}, W(\cdot)$ be a standard $\mathcal{F}_{t}$-Wiener process, and $m(\cdot)$ be admissible. Let $\widehat{\mathcal{F}}_{t}$ be the $\sigma$-algebra generated by $\left\{\widehat{\xi}^{h}(s), \widehat{\alpha}^{h}(s), \widehat{m}^{h}(s), \widehat{W}^{h}(s), \widehat{N}^{h}(s), \widehat{R}^{h}(s), \widehat{z}^{h}(s)\right.$, $\left.\widehat{l}^{h}(s), \widehat{T}^{h}(s): s \leq t\right\}$. Then $\widehat{W}(t)=W(\widehat{T}(t))$ is an $\widehat{\mathcal{F}}_{t}$-martingale with quadratic variation $\widehat{T}(t)$. The limit processes satisfy

$$
\begin{aligned}
\widehat{X}(t)= & x+\int_{0}^{t} \int_{\mathcal{U}}[g(\widehat{\alpha}(s))(1-\psi)+\psi b(\widehat{\alpha}(s))] \widehat{X}(s) \\
& +c(\widehat{\alpha}(s))] \widehat{m} \widehat{T}(s) \\
& +\int_{0}^{t} \int_{\mathcal{U}} \psi \sigma(\widehat{\alpha}(s)) \widehat{X}(s) \widehat{M} \widehat{T}(s) \\
& -\widehat{R}(t)-\widehat{z}(t)-\widehat{l}(t) .
\end{aligned}
$$

Theorem 8 Under the conditions of Theorem 7 , for $t<$ $\infty$, define the inverse $\bar{T}(t)=\inf \{s: \widehat{T}(s)>t\}$. Then $\bar{T}(t)$ is right continuous and $\bar{T}(t) \rightarrow \infty$ as $t \rightarrow \infty$ w.p. 1 .
For any process $\widehat{\chi}(\cdot)$, define the rescaled process $\chi(\cdot)$ by $\chi(t)=\widehat{\chi}(\bar{T}(t))$. Then, $W(\cdot)$ is a standard $\mathcal{F}_{t}$-Wiener process, $N(\cdot)$ is a Poisson measure and (2.3) holds.

Proof. Since $\widehat{T}(t) \rightarrow \infty$ w.p. 1 as $t \rightarrow \infty, \bar{T}(t)$ exists for all $t$ and $\bar{T}(t) \rightarrow \infty$ as $t \rightarrow \infty$ w.p.1. Similar to (A.5) and (A.7), for each $i \in \mathcal{M}$,

$$
\begin{aligned}
& E \Phi\left(\xi^{h}\left(t_{k}\right), \alpha^{h}\left(t_{k}\right), W^{h}\left(t_{k}\right), N^{h}\left(t_{k}, \Gamma_{j}^{\kappa}\right),\left(\chi_{j}, m^{h}\right)_{t_{k}},\right. \\
& \left.\quad R^{h}\left(t_{k}\right), z^{h}\left(t_{k}\right), l^{h}\left(t_{k}\right), j \leq \kappa, k \leq p\right) \\
& \quad \times[W(t+s)-W(t)]=0 . \\
& E \Phi\left(\xi^{h}\left(t_{k}\right), \alpha^{h}\left(t_{k}\right), W^{h}\left(t_{k}\right), N^{h}\left(t_{k}, \Gamma_{j}^{\kappa}\right),\left(\chi_{j}, m^{h}\right)_{t_{k}},\right. \\
& \left.\quad R^{h}\left(t_{k}\right), z^{h}\left(t_{k}\right), l^{h}\left(t_{k}\right), j \leq \kappa, k \leq p\right) \\
& \quad \times\left[W^{2}(t+s)-W^{2}(t)-(\bar{T}(t+s)-\bar{T}(t))\right]=0 .
\end{aligned}
$$

Thus, we can verify $W(\cdot)$ is an $\mathcal{F}_{t}$-Wiener process. A rescaling of (4.20) yields

$$
\begin{aligned}
X(t)=x & +\int_{0}^{t} \int_{\mathcal{U}}[[g(\alpha(s))(1-\psi)+\psi b(\alpha(s))] X(s) \\
& +c(\alpha(s))] m_{s}(d \psi) d s \\
& +\int_{0}^{t} \int_{\mathcal{U}} \psi \sigma(\alpha(s)) X(s) M_{s}(d \psi) d s-R(t) \\
& -z(t)-l(t) .
\end{aligned}
$$

In other words, (2.3) holds.

\subsection{A Chattering Lemma and Approximation to the Op- timal Control}

We consider the approximation of relaxed controls by ordinary controls in this subsection. Here we present a result of chattering lemma for our problem. The proof of the chattering lemma can be found in [Kushner, 1990].

Lemma 9 Assume the conditions of Theorem 7. Let $(m(\cdot), W(\cdot))$ be admissible for the problem given in (4.14). Then given $\varrho>0$, there is a finite set $\left\{\gamma_{1}^{\varrho}, \ldots, \gamma_{l_{o}}^{\varrho}\right\}=$ $U^{\varrho} \subset U$, and an $\varepsilon>0$ such that there is a probability space on which are defined $\left(X^{\varrho}(\cdot), \alpha(\cdot), u^{\varrho}(\cdot), W^{\varrho}(\cdot)\right.$, $\left.N^{\varrho}(\cdot)\right)$, where $W^{\varrho}(\cdot)$ and $N^{\varrho}(\cdot)$ are standard Brownian motions and Poisson measure, and $u^{\varrho}(\cdot)$ is an admissible $U^{\varrho}$-valued ordinary control on the interval $[k \varepsilon, k \varepsilon+\varepsilon)$. Moreover,

$$
P_{x}^{m}\left(\sup _{s \leq T}\left|X^{\varrho}(s)-X(s)\right|>\varrho\right) \leq \varrho .
$$

Coming back to the approximation to the optimal control, to show $V^{h}(x, i)$ converges to $V(x, i)$, we shall use 
the comparison control techniques. Certain continuity properties need to be verified as following.

Lemma 10 Under the conditions of Lemma 9, for each $\varrho>0$, there is an $\varepsilon>0$ and a probability space on which are defined $W^{\varrho}(\cdot)$, a control $u^{\varrho}(\cdot)$ as in Lemma 9 , and a solution $X^{\varrho}(\cdot)$ such that the following assertions hold:

(i)

$$
\left|J^{m}(x, i, \cdot)-J^{u^{\varrho}}(x, i, \cdot)\right| \leq \varrho, \text { for } i \in \mathcal{M}
$$

(ii) Moreover, there is a $\theta>0$ and a partition $\left\{\Gamma_{j}^{\kappa}, j \leq \kappa\right\}$ of $\Gamma$ such that the approximating $u^{\varrho}(\cdot)$ can be chosen so that its probability law at $n \varepsilon$, conditioned on $\left\{W^{\varrho}(\tau), \alpha(\tau), N^{\varrho}(\tau), \tau \leq n \varepsilon ; u^{\varrho}(k \varepsilon), k<n\right\}$ depends only on the samples $\left\{\bar{W}^{\varrho}(p \theta), \alpha(p \theta), N^{\varrho}(p \theta), p \theta \leq\right.$ $\left.n \varepsilon ; u^{\varrho}(k \varepsilon), k<n\right\}$, and is continuous in the $W^{\varrho}(p \bar{\theta})$ arguments.

\subsection{Convergence of Cost and Value Functions}

Theorem 11 Assume the conditions of Theorem 7, Theorem 8, Lemma 9, and Lemma 10 are satisfied. Then as $h \rightarrow 0$,

$$
\begin{aligned}
& J^{h}\left(x, i, \pi^{h}\right) \rightarrow E_{x, i}^{\pi} \int_{0}^{\infty} e^{-r t} \beta_{1} d z \\
& \quad-\sum_{n=1}^{\infty} e^{-\delta \tau_{n}}\left(K+\beta_{2} \zeta_{n}\right) I_{\left\{\tau_{n}<\infty\right\}}=J(x, i, \pi) .
\end{aligned}
$$

Proof. Note that $\Delta z^{h}=h$, the uniform integrability of $d z$ can be easily verified. Due to the tightness and the uniform integrability properties, for any $t, \int_{0}^{t} d \widehat{z}$ can be well approximated by a Reimann sum uniformly in $h$. By the weak convergence and the Skorohod representation,

$$
E_{x, i}^{\pi} \sum_{k=1}^{\infty} e^{-r t_{k}^{h}} \beta_{1} \Delta z_{k}^{h} \rightarrow E_{x, i}^{\pi} \int_{0}^{\infty} e^{-r \widehat{T}(t)} \beta_{1} d \widehat{z} .
$$

By an inverse transformation,

$$
E_{x, i}^{\pi} \int_{0}^{\infty} e^{-r \widehat{T}(t)} \beta_{1} d \widehat{z}=E_{x, i}^{\pi} \int_{0}^{\infty} e^{-r t} \beta_{1} d z
$$

Also,

$$
\begin{aligned}
& E_{x, i}^{\pi} \sum_{k=1}^{\infty} e^{-r t_{k}^{h}}\left(\beta_{2} \Delta l_{k}^{h}+K\right) \\
& \rightarrow E_{x, i}^{\pi} \sum_{n=1}^{\infty} e^{-\delta \tau_{n}}\left(\beta_{2} \zeta_{n}+K\right) I_{\left\{\tau_{n}<\infty\right\}} .
\end{aligned}
$$

Thus, as $h \rightarrow 0$,

$$
J^{h}\left(x, i, \pi^{h}\right) \rightarrow J(x, i, \pi) .
$$

Theorem 12 Assume that conditions of Theorem 11 are satisfied. For $V^{h}(x, i)$ and $V(x, i)$, value functions defined in (4.16) and (2.5), respectively, we have $V^{h}(x, i) \rightarrow V(x, i)$ as $h \rightarrow 0$.

The proof of this theorem is given in the appendix.

\section{$5 \quad$ Numerical Examples}

This section is devoted to several examples. For simplicity, we consider the case that the discrete event has two states. That is, the continuous-time Markov chain has two states with given claim size distributions. By using value iteration methods, we numerically solve the optimal control problems. We approximate the value functions and optimal controls in exponential claim severity distributions in which the tail of distribution is considered light. Exponential distribution is applicable for automobile losses. The corresponding capital injection sizes and barriers for regions are also obtained in the numerical examples.

Based on the algorithm constructed above, we carry out the computation by value iterations. For $n \in Z^{+}$and $i \in \mathcal{M}$, define the vectors

$$
\begin{aligned}
& V_{n}^{h}=\left\{V_{n}^{h}(h, 1), V_{n}^{h}(2 h, 1), \ldots, V_{n}^{h}(B, 1), \ldots V_{n}^{h}\left(h, n_{0}\right),\right. \\
& \left.\quad V_{n}^{h}\left(2 h, n_{0}\right), \ldots, V_{n}^{h}\left(B, n_{0}\right)\right\} \\
& V^{h}=\left\{V_{n}(h, 1), V_{n}(2 h, 1), \ldots, V_{n}(B, 1), \ldots V_{n}\left(h, n_{0}\right),\right. \\
& \left.\quad V_{n}\left(2 h, n_{0}\right), \ldots, V_{n}\left(B, n_{0}\right)\right\} .
\end{aligned}
$$

Using the method of value iteration, we obtain $V_{n}^{h} \rightarrow V^{h}$ as $n \rightarrow \infty$.

1. Set $n=0 . \forall x \in S_{h}$ and $i \in \mathcal{M}$, we set the initial value $V^{h}(x, i)=1$.

2. Find improved values $V_{n+1}^{h}(x, i)$ by (3.16) and record the corresponding optimal control.

$$
\begin{aligned}
& V_{n+1}^{h}(x, i) \\
& =\max _{\pi \in \mathcal{A}}\left[\left(1-\lambda \Delta t^{h}(x, i, u, 2)+\delta^{h}(x, i, u, 2)\right)\right. \\
& \times e^{-r \Delta t^{h}(x, i, u, 2)} \sum_{(y, j)}\left(p_{D}^{h}((x, i),(y, j)) \mid \pi\right) V^{h}(y, i) \\
& +\left(\lambda \Delta t^{h}(x, i, u, 2)+\delta^{h}(x, i, u, 2)\right) e^{-r \Delta t^{h}(x, i, u, 2)} \\
& \times \int_{0}^{x} V_{n}^{h}(x-y, i) \Pi(d y), \\
& \left.V_{n}^{h}(x-h, i)+\beta_{1} h, \sup _{0 \leq \widetilde{y} \leq B-x} V_{n}^{h}(x+\widetilde{y}, i)-\beta_{2} \widetilde{y}-K\right]
\end{aligned}
$$




$$
\begin{aligned}
& \pi_{n+1}^{h}(x, i) \\
& =\underset{\pi \in \mathcal{A}}{\operatorname{argmax}}\left[\left(1-\lambda \Delta t^{h}(x, i, u, 2)+\delta^{h}(x, i, u, 2)\right)\right. \\
& \times e^{-r \Delta t^{h}(x, i, u, 2)} \sum_{(y, j)}\left(p_{D}^{h}((x, i),(y, j)) \mid \pi\right) V^{h}(y, i) \\
& +\left(\lambda \Delta t^{h}(x, i, u, 2)+\delta^{h}(x, i, u, 2)\right) e^{-r \Delta t^{h}(x, i, u, 2)} \\
& \times \int_{0}^{x} V_{n}^{h}(x-y, i) \Pi(d y), \\
& \left.V_{n}^{h}(x-h, i)+\beta_{1} h, \underset{0 \leq \widetilde{y} \leq B-x}{\sup } V_{n}^{h}(x+\widetilde{y}, i)-\beta_{2} \widetilde{y}-K\right]
\end{aligned}
$$

3. If $\left|V_{n+1}^{h}-V_{n}^{h}\right|>$ tolerance, then $n \rightarrow n+1$ and go to step 2; else the iteration stops.

Example 13 The continuous-time Markov chain $\alpha(t)$ representing the discrete event state has the generator $Q=\left(\begin{array}{cc}-0.5 & 0.5 \\ 0.5 & -0.5\end{array}\right)$, and takes values in $\mathcal{M}=\{1,2\}$. The claim severity distribution follows exponential distribution with density function $f(y)=a e^{-a y}$ where $a=0.1$. Furthermore, $\left\{\nu_{n+1}-\nu_{n}\right\}$ is a sequence of exponentially distributed random variables with mean 10 . Then $\lambda=0.1$. The premium rate depends on the discrete state with $c(1)=2$ and $c(2)=10$. The portfolio rate $u(t)$ taking values in $[0,1]$ is the control. Corresponding to the different discrete states, the yield rate of the riskless asset is $g(1)=0.03$ and $g(2)=0.04$, whereas the risky asset return rate is $b(1)=0.06$ and $b(2)=0.08$. The volatility of the financial market $\sigma(\alpha(t))$ is valued as $\sigma(1)=0.1$ and $\sigma(2)=0.2$. Let the upper bound of the computation interval $B=100$, the discount rate $r=0.05$, the fixed capital injection cost $K=1$, the parameters for the proportion costs of dividend payments and capital injections $\beta_{1}=0.9, \beta_{2}=1.1$.

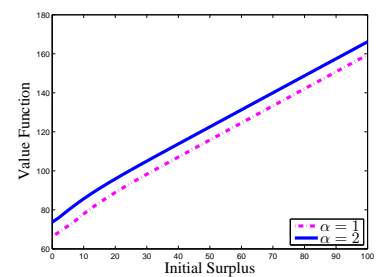

Fig. 5.2. Optimal value function versus surplus process

All the figures contain two curves since we consider the two-regime case. Figure 5.2 shows that the value functions are concave and increase with slope $\beta_{1}$ after certain barrier, which means the extra surplus will all be paid out as the dividend after reaching sufficient high threshold. In addition, because of the availability of immediate capital injections, the initial value $V(0)$ is nonzero because the capital injections can always guarantee the continuity of the business even with the zero initial surplus. Hence, financial ruin can be avoided. Moreover, the total expected discounted value of all dividends could lead to infinity, since this result does not obey the assumption that the discount rate is higher than the maximal yield rate. Otherwise, the total expected discounted value of all dividends will be bounded.

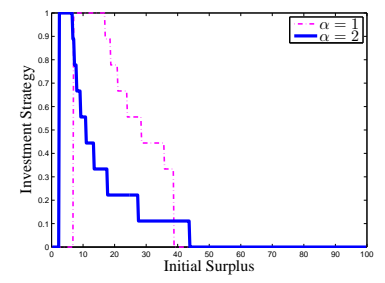

Fig. 5.3. Optimal investment strategy versus initial surplus

Regarding the investment strategy, we observe from Figure 5.3 that the proportion of investment in the risky asset will be zero after certain threshold. To maximize the total expected discounted value of all the dividends, the rational insurers seem to be risk averse. The decision makers choose to lower the proportion of risky assets gradually as the surplus increases. When surplus is higher than certain barrier, all of the surplus will be invested in the risk free asset. This is a natural investment strategy since insurance company actually holds large stakes of bonds to guarantee the finance safety for a promise of repayment. In addition, the insurer prefers to put big weight money in the risky asset when the surplus is not high enough. At the mean time, the optimal discounted dividend increases with a faster pace (the derivative is greater than 1). In other words, with small amount of money, the rational investor makes the investment more efficient by choosing investment strategy aggressively. Furthermore, from the two lines in the graphs, it is shown that the investment strategy varies in different regimes due to the Markov switching.

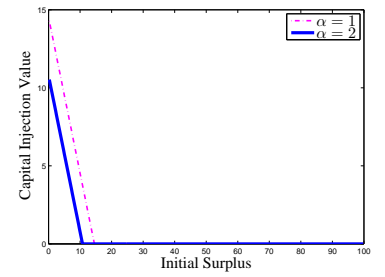

Fig. 5.4. Optimal capital injection sizes versus initial surplus

Figure 5.4 provides the relationship between the optimal capital injection size and the initial surplus. It shows that the capital injection size decreases with the increase of the surplus. That is, healthier financial condition needs less capital injections, which is in accordance with the intuitive thinking. The state-dependent capital injection sizes approaches zero when surplus hits certain barrier. The capital injection becomes unnecessary until the surplus pass the barrier, which means that the 


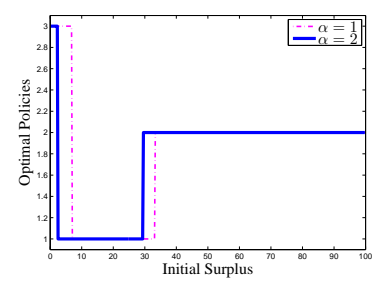

Fig. 5.5. Regions versus initial surplus

company possesses sufficient capital to guarantee the financial safety.

In Figure 5.5, we use " 1 " to denote the region in the QVIs when regular control is dominant, "2" to denote the region in the QVIs when dividend payment is dominant, and "3" to denote the region in the QVIs when capital injections are dominant. We found that regimeswitchings have obvious impact on the optimal strategies and the barriers of the regions. Not only the optimal values of the discounted total dividend in different regimes have big difference, but also the dividend payment policies are very different in different regimes. In particular, we observe that the dividend payment is dominant when the investment in risky assets becomes zero. It seems the insurer chooses to put money in the riskless asset or pay out the surplus as dividend when it is high enough to avoid the possible risk. Moreover, with the sufficient low surplus, capital injections are optimal and the investment is preferred in risky assets.

\section{Concluding Remarks}

In this work, we have developed numerical approximation schemes for finding the optimal investment and dividend payment policy to maximize the total discounted dividend payments less the possible capital injections until the lifetime of ruin. A generalized regime-switching jump diffusion formulation of surplus with capital injections is presented. Although one could derive the associated system of integro-differential QVIs by using the usual dynamic programming approach together with the use of properties of regime-switchings, solving the investment problem with singular and impulse controls analytically is very difficult. As an alternative, we presented a Markov chain approximation method using mainly probabilistic methods. For the singular and impulse control part, a technique of time rescaling is used. In the actual computation, the optimal value function can be obtained by using the value iterations or policy iterations. For further study, time delays of the capital injections can be considered. It is intuitive to analyze the more realistic capital injections, where time delay occurs due to the regulatory process. In the real world, the capital injection can never happen instantaneously. Time delays cannot be ignored and are unavoidable so stochastic delayed system will be more realistic and more complicated. It is virtually impossible to obtain a closed-form solution. Numerical approximation can provide a viable alternative.

\section{A Appendix}

\section{A.1 Proof of Theorem 7}

Proof. For $\delta>0$, define the process $f(\cdot)$ by $f^{h, \delta}(t)=$ $f^{h}(n \delta), t \in[n \delta,(n+1) \delta)$. Then, by the tightness of $\left\{\widehat{\xi}^{h}(\cdot), \widehat{\alpha}^{h}(\cdot)\right\},(4.15)$ can be rewritten as

$$
\begin{aligned}
\widehat{\xi}^{h}(t)=x & +\int_{0}^{t} \int_{\mathcal{U}}\left[\left[g\left(\widehat{\alpha}^{h}(s)\right)(1-\psi)+\psi b\left(\widehat{\alpha}^{h}(s)\right)\right] \widehat{\xi}^{h}(s)\right. \\
& \left.+c\left(\widehat{\alpha}^{h}(s)\right)\right] \widehat{m}_{\widehat{T}^{h}(s)}^{h}(d \psi) d \widehat{T}^{h}(s) \\
& +\int_{0}^{t} \int_{\mathcal{U}} \psi \sigma\left(\widehat{\alpha}^{h, \delta}(s)\right) \widehat{\xi}^{h, \delta}(s) \widehat{M}_{\widehat{T}^{h}(s)}(d \psi) d \widehat{T}^{h}(s) \\
& -\widehat{R}^{h}(t)-\widehat{z}^{h}(t)-\widehat{l}^{h}(t)+\varepsilon^{h, \delta}(t),
\end{aligned}
$$

where

$$
\lim _{\delta \rightarrow 0} \limsup _{h \rightarrow 0} E\left|\varepsilon^{h, \delta}(t)\right|=0 .
$$

If we can verify that $\widehat{W}(\cdot)$ is an $\widehat{\mathcal{F}}_{t}$-martingale, then (4.20) could be obtained by taking limits in (A.1). To characterize $W(\cdot)$, let $t>0, \delta>0, p, \kappa,\left\{t_{k}: k \leq p\right\}$ be given such that $t_{k} \leq t \leq t+\delta$ for all $k \leq p, \phi_{j}(\cdot)$ for $j \leq \kappa$ is real-valued and continuous functions on $U \times[0, \infty)$ having compact support for all $j \leq q$. Define

$$
\left(\phi_{j}, \widehat{m}\right)_{t}=\int_{0}^{t} \int_{\mathcal{U}} \phi_{j}(\psi, s) \widehat{m}_{\widehat{T}(s)}(d \psi) d \widehat{T}(s) .
$$

Let $\left\{\Gamma_{j}^{\kappa}, j \leq \kappa\right\}$ be a sequence of nondecreasing partition of $\Gamma$ such that $\Pi\left(\partial \Gamma_{j}^{\kappa}\right)=0$ for all $j$ and all $\kappa$, where $\partial \Gamma_{j}^{\kappa}$ is the boundary of the set $\Gamma_{j}^{\kappa}$. As $\kappa \rightarrow \infty$, let the diameter of the sets $\Gamma_{j}^{\kappa}$ go to zero. Let $\Phi(\cdot)$ be a realvalued and continuous function of its arguments with compact support. In view of the definition of $\widehat{W}(t)$, for each $i \in \mathcal{M}$, we have

$$
\begin{aligned}
& E \Phi\left(\widehat{\xi}^{h}\left(t_{k}\right), \widehat{\alpha}^{h}\left(t_{k}\right), \widehat{W}^{h}\left(t_{k}\right), \widehat{N}^{h}\left(t_{k}, \Gamma_{j}^{\kappa}\right),\left(\phi_{j}, m^{h}\right)_{t_{k}},\right. \\
& \left.\widehat{R}^{h}\left(t_{k}\right), \widehat{z}^{h}\left(t_{k}\right), \widehat{l}^{h}\left(t_{k}\right), j \leq \kappa, k \leq p\right) \\
& \times\left[\widehat{W}^{h}(t+\delta)-\widehat{W}^{h}(t)\right]=0 .
\end{aligned}
$$

By using the Skorohod representation and the dominant convergence theorem, letting $h \rightarrow 0$, we obtain

$$
\begin{aligned}
E & \Phi\left(\widehat{\xi}^{h}\left(t_{k}\right), \widehat{\alpha}^{h}\left(t_{k}\right), \widehat{W}^{h}\left(t_{k}\right), \widehat{N}^{h}\left(t_{k}, \Gamma_{j}^{\kappa}\right),\left(\phi_{j}, m^{h}\right)_{t_{k}},\right. \\
& \left.\widehat{R}^{h}\left(t_{k}\right), \widehat{z}^{h}\left(t_{k}\right), \widehat{l}^{h}\left(t_{k}\right), j \leq \kappa, k \leq p\right) \\
& \times[\widehat{W}(t+\delta)-\widehat{W}(t)]=0 .
\end{aligned}
$$


Since $\widehat{W}(\cdot)$ has continuous sample paths, (A.5) implies that $\widehat{W}(\cdot)$ is a continuous $\mathcal{F}_{t}$-martingale. On the other hand, since

$$
\begin{aligned}
& E\left[\left(\left(\widehat{W}^{h}(t+\delta)\right)^{2}-\left(\widehat{W}^{h}(t)\right)^{2}\right]\right. \\
& =E\left[\left(\widehat{W}^{h}(t+\delta)-\widehat{W}^{h}(t)\right)^{2}\right] \\
& =\widehat{T}(t+\delta)-\widehat{T}(t),
\end{aligned}
$$

by using the Skorohod representation and the dominant convergence theorem together with (A.6), we have

$$
\begin{aligned}
& E \Phi\left(\widehat{\xi}^{h}\left(t_{k}\right), \widehat{\alpha}^{h}\left(t_{k}\right), \widehat{W}^{h}\left(t_{k}\right), \widehat{N}^{h}\left(t_{k}, \Gamma_{j}^{\kappa}\right),\left(\phi_{j}, m^{h}\right)_{t_{k}},\right. \\
& \left.\quad \widehat{R}^{h}\left(t_{k}\right), \widehat{z}^{h}\left(t_{k}\right), \widehat{l}^{h}\left(t_{k}\right), j \leq \kappa, k \leq p\right) \\
& \quad \times\left[\widehat{W}^{2}(t+\delta)-\widehat{W}^{2}(t)-(\widehat{T}(t+\delta)-\widehat{T}(t))\right]=0 .
\end{aligned}
$$

The quadratic variation of the martingale $\widehat{W}(t)$ is $\Delta \widehat{T}$, then $\widehat{W}(\cdot)$ is an $\widehat{\mathcal{F}}_{t}$-Wiener process.

Let $h \rightarrow 0$, by using the Skorohod representation, we obtain

$$
\begin{aligned}
& E \mid \int_{0}^{t} \int_{\mathcal{U}}\left[\left[g\left(\widehat{\alpha}^{h}(s)\right)(1-\psi)+\psi b\left(\widehat{\alpha}^{h}(s)\right)\right] \widehat{\xi}^{h}(s)\right. \\
& \left.\quad+c\left(\widehat{\alpha}^{h}(s)\right)\right] \widehat{m}_{\widehat{T}^{h}(s)}^{h}(d \psi) d \widehat{T}^{h}(s) \\
& \quad-\int_{0}^{t} \int_{\mathcal{U}}[[g(\widehat{\alpha}(s))(1-\psi)+\psi b(\widehat{\alpha}(s))] \widehat{X}(s) \\
& \quad+c(\widehat{\alpha}(s))] \widehat{m}_{\widehat{T}(s)}^{h}(d \psi) d \widehat{T}^{h}(s) \mid \rightarrow 0
\end{aligned}
$$

uniformly in $t$. On the other hand, $\left\{\widehat{m}^{h}(\cdot)\right\}$ converges in the compact weak topology, that is, for any bounded and continuous function $\phi(\cdot)$ with compact support, as $h \rightarrow 0$,

$$
\begin{aligned}
\int_{0}^{\infty} \int_{\mathcal{U}} \phi(\psi, s) \widehat{m}_{\widehat{T}^{h}(s)}^{h}(d \psi) d \widehat{T}^{h}(s) \\
\quad \rightarrow \int_{0}^{\infty} \int_{\mathcal{U}} \phi(\psi, s) \widehat{m}_{\widehat{T}(s)}(d \psi) d \widehat{T}(s)
\end{aligned}
$$

Again, the Skorohod representation (with a slight abuse of notation) implies that as $h \rightarrow 0$,

$$
\begin{aligned}
& \int_{0}^{t} \int_{\mathcal{U}}\left[\left[g\left(\widehat{\alpha}^{h}(s)\right)(1-\psi)+\psi b\left(\widehat{\alpha}^{h}(s)\right)\right] \widehat{\xi}^{h}(s)\right. \\
& \left.\quad+c\left(\widehat{\alpha}^{h}(s)\right)\right] \widehat{m}_{\widehat{T}^{h}(s)}^{h}(d \psi) d \widehat{T}^{h}(s) \\
& \quad \rightarrow \int_{0}^{t} \int_{\mathcal{U}^{\prime}}[[g(\widehat{\alpha}(s))(1-\psi)+\psi b(\widehat{\alpha}(s))] \widehat{X}(s) \\
& \quad+c(\widehat{\alpha}(s))] \widehat{m} \widehat{T}(s)
\end{aligned}
$$

uniformly in $t$ on any bounded interval.
In view of $(\mathrm{A} .1)$, since $\xi^{h, \delta}(\cdot)$ and $\alpha^{h, \delta}(\cdot)$ are piecewise constant functions,

$$
\begin{aligned}
\int_{0}^{t} & \int_{\mathcal{U}} \psi \sigma\left(\widehat{\alpha}^{h, \delta}(s)\right) \widehat{\xi}^{h, \delta}(s) \widehat{M}_{\widehat{T}^{h}(s)}(d \psi) d \widehat{T}^{h}(s) \\
\rightarrow & \int_{0}^{t} \int_{\mathcal{U}} \psi \sigma\left(\widehat{\alpha}^{\delta}(s)\right) \widehat{\xi}^{\delta}(s) \widehat{M}_{\widehat{T}(s)}(d \psi) d \widehat{T}(s)
\end{aligned}
$$

as $h \rightarrow 0$. Combining (A.3)-(A.11), we have

$$
\begin{aligned}
\widehat{X}(t)= & x+\int_{0}^{t} \int_{\mathcal{U}}[[g(\widehat{\alpha}(s))(1-\psi)+\psi b(\widehat{\alpha}(s))] \widehat{X}(s) \\
& +c(\widehat{\alpha}(s))] \widehat{m_{\widehat{T}}(s)}(d \psi) d \widehat{T}(s) \\
& +\int_{0}^{t} \int_{\mathcal{U}} \psi \sigma\left(\widehat{\alpha}^{\delta}(s)\right) \widehat{X}^{\delta}(s) \widehat{M}_{\widehat{T}(s)}(d \psi) d \widehat{T}(s)-\widehat{R}(t) \\
& -\widehat{z}(t)-\widehat{l}(t)+\varepsilon^{\delta}(t)
\end{aligned}
$$

where $\lim _{\delta \rightarrow 0} E\left|\varepsilon^{\delta}(t)\right|=0$. Finally, taking limits in the above equation as $\delta \rightarrow 0,(4.20)$ is obtained.

\section{A.2 Proof of Theorem 12}

Proof. First, to prove

$$
V(x, i) \geq \limsup _{h} V^{h}(x, i) .
$$

Since $V(x, i)$ is the maximizing cost function, for any admissible control $\pi(\cdot), J(x, i, \pi) \leq V(x, i)$. Let $\widetilde{m}^{h}(\cdot)$ be an optimal relaxed control for $\left\{\xi^{h}(\cdot)\right\}$ and $\widetilde{\pi}^{h}(\cdot)=$ $\left(\widetilde{m}^{h}(\cdot), \widetilde{z}^{h}(\cdot), \widetilde{l}^{h}(\cdot)\right)$. That is, $V^{h}(x, i)=J^{h}\left(x, i, \widetilde{\pi}^{h}\right)$ $=\sup _{\pi^{h}} J^{h}\left(x, i, \pi^{h}\right)$. Choose a subsequence $\{\widetilde{h}\}$ of $\{h\}$ such that $\lim _{\widetilde{h} \rightarrow 0} V^{\widetilde{h}}(x, i)=\limsup _{\widetilde{h} \rightarrow 0} V^{\widetilde{h}}(x, i)=$ $\lim _{\widetilde{h} \rightarrow 0} J^{\widetilde{h}}\left(x, i, \widetilde{\pi}^{h}\right)$. Without loss of generality (passing to an additional subsequence if needed), we may assume that $\left(\xi^{\widetilde{h}}(\cdot), \alpha^{\widetilde{h}}(\cdot), m^{\widetilde{h}}(\cdot), W^{\widetilde{h}}(\cdot), N^{\widetilde{h}}(\cdot), R^{\widetilde{h}}(\cdot), z^{\widetilde{h}}(\cdot), l^{\widetilde{h}}(\cdot)\right)$ converges weakly to $(X(\cdot), \alpha(\cdot), m(\cdot), W(\cdot), N(\cdot)$, $R(\cdot), z(\cdot), l(\cdot))$, where $\pi(\cdot)$ is an admissible related control. Then the weak convergence and the Skorohod representation yield that

$$
\underset{h}{\limsup } V^{h}(x, i)=J(x, i, \pi) \leq V(x, i) .
$$

We proceed to prove the reverse inequality.

We claim that

$$
V(x, i) \leq \liminf _{h} V^{h}(x, i) .
$$

Suppose that $\bar{m}$ is an optimal control with Brownian motion $W(\cdot)$ such that $\bar{X}(\cdot)$ is the associated trajectory and $\bar{\pi}(\cdot)=(\bar{m}(\cdot), \bar{z}(\cdot), \bar{l}(\cdot))$. By the chattering lemma, given 
any $\epsilon>0$, there are an $\varepsilon>0$ and an ordinary control $u^{\epsilon}(\cdot)$ that takes only finite many values, that $u^{\epsilon}(\cdot)$ is a constant on $[k \varepsilon, k \varepsilon+\varepsilon) . z^{\epsilon}(\cdot)$ and $l^{\epsilon}(\cdot)$ are defined analogously. $\bar{m}^{\epsilon}(\cdot)$ is the relaxed control representation, and $\left(\bar{X}^{\epsilon}(\cdot), \bar{m}^{\epsilon}(\cdot)\right)$ converges weakly to $(X(\cdot), \bar{m}(\cdot))$. Then $J\left(x, i, \bar{\pi}^{\epsilon}\right) \geq V(x, i)-\epsilon$.

For each $\epsilon>0$, and the corresponding $\varepsilon>0$ as in the chattering lemma, consider an optimal control problem as in (2.3) with piecewise constant on $[k \varepsilon, k \varepsilon+\varepsilon)$. For this controlled diffusion process, we consider its $\epsilon$-skeleton. By that we mean we consider the process $\left(X^{\epsilon}(k \varepsilon), m^{\epsilon}(k \varepsilon)\right)$. Let $\tilde{u}^{\epsilon}(\cdot)$ be the optimal control, $\tilde{m}^{\epsilon}(\cdot)$ the relaxed control representation and $\tilde{\pi}^{\epsilon}(\cdot)=\left(\tilde{m}^{\epsilon}(\cdot), \tilde{z}^{\epsilon}(\cdot), \tilde{l}^{\epsilon}(\cdot)\right)$, and $\tilde{X}^{\epsilon}(\cdot)$ the associated trajectory. Since $\tilde{m}^{\epsilon}(\cdot)$ is optimal control, $J\left(x, i, \tilde{\pi}^{\epsilon}\right) \geq J\left(x, i, \bar{\pi}^{\epsilon}\right) \geq V(x, i)-\epsilon$. We next approximate $\tilde{u}^{\epsilon}(\cdot)$ by a suitable function of $(W(\cdot), \alpha(\cdot))$. Moreover, $V^{h}(x, i) \geq J^{h}\left(x, i, \bar{\pi}^{h}\right) \rightarrow J\left(x, i, \bar{\pi}^{\epsilon, \theta}\right)$ Thus,

$$
\liminf _{h} V^{h}(x, i) \geq J^{h}\left(x, i, \bar{\pi}^{h}\right) \rightarrow J\left(x, i, \bar{\pi}^{\epsilon, \theta}\right) .
$$

Using the result obtained in Proposition 10,

$$
\liminf _{h} V^{h}(x, i) \geq V(x, i)-2 \epsilon .
$$

The arbitrariness of $\epsilon$ then implies that $\liminf _{h} V^{h}(x, i) \geq$ $V(x, i)$.

Using (A.14) and (A.15) together with the weak convergence and the Skorohod representation, we obtain the desired result. The proof of the theorem is concluded. $\square$

\section{References}

[Azcue and Muler, 2010] Azcue, P. and Muler, N. (2010). Optimal investment policy and dividend payment strategy in an insurance company. The Annals of Applied Probability, 20(4): 1253-1302.

[Asmussen and Taksar, 1997] Asmussen, S. and Taksar M. (1997). Controlled diffusion models for optimal dividend pay-out. Insurance: Math. and Economics, 20: 1-15.

[Browne, 1995] Browne, S. (1995). Optimal investment policies for a firm with a random risk process: Exponential utility and minimizing the probability of ruin. Mathematics of Operations Research 20(4): 937958.

[Budhiraja and Ross, 2007] Budhiraja, A. and Ross, K. (2007). Convergent numerical scheme for singular stochastic control with state constraints in a portfolio selection problem. SIAM J. Control Optim., 45(6): 2169 2206.

[Dickson and Waters, 2004] Dickson, DCM, Waters, HR (2004). Some optimal dividends problems. ASTIN Bulletin 34(1):49-74.

[De Finetti, 1957] De

Finetti, B. (1957). Su unimpostazione alternativa della teoria collettiva del rischio, Transactions of the XVth International Congress of Actuaries, 2 (1957): 433-443.
[Fleming and Soner, 2006] Fleming, W. and Soner, H. (2006). Controlled Markov Processes and Viscosity Solutions, volume 25 of Stochastic Modelling and Applied Probability. Springer-Verlag, New York, NY, second edition.

[Gerber and Shiu, 2004] Gerber, H. and Shiu, E. (2004). Optimal dividends: analysis with Brownian motion. North American Actuarial Journal, 8: 1-20.

[Jin et al., 2012] Jin, Z., Yin, G., and Zhu, C. (2012). Numerical solutions of optimal risk control and dividend optimization policies under a generalized singular control formulation, Automatica 48(8): 1489-1501.

[Kulenko and Schimidli, 2008] Kulenko, N. and Schimidli, H. (2008). An optimal dividend strategy in a Craḿer Lundberg model with capital injections. Insurance: Mathmatics and Economics, 43: 270-278.

[Kushner, 1990] Kushner, H. (1990). Weak Convergence Methods and Singularly Perturbed Stochastic Control and Filtering Problems, Birkhäuser, Boston, MA.

[Kushner and Dupuis, 2001] Kushner, H. and Dupuis, P. (2001). Numerical Methods for Stochstic Control Problems in Continuous Time, volume 24 of Stochastic Modelling and Applied Probability. Springer, New York, second edition.

[Kushner and Martins, 1991] Kushner, H. J. and Martins, L. F. (1991). Numerical methods for stochastic singular control problems. SIAM J. Control Optim., 29: 14431475.

[Hamilton, 1989] Hamilton, J. (1989). A new approach to the economic analysis of non-stationary time series. Econometrica, (57): 357-384.

[Sethi and Taksar, 2002] Sethi, S.P. and Taksar, M. (2002). Optimal financing of a corporation subject to random returns. Mathematical Finance, 12: 155-172.

[Sotomayor and Cadenillas, 2011] Sotomayor, L. and Cadenillas, A. (2011). Classical, singular, and impulse stochastic control for the optimal dividend policy when there is regime switching. Insurance: Mathematics and Economics, 48(3): 344-354

[Yang and Yin, 2004] Yang, H. and Yin, G. (2004). Ruin probability for a model under Markovian switching regime, In T.L. Lai, H. Yang, and S.P. Yung, editors, Probability, Finance and Insurance, pages 206-217. World Scientific, River Edge, NJ.

[Yao et al., 2011] Yao, D., Yang, H. and Wang, R. (2011). Optimal dividend and capital injection problem in the dual model with proportional and fixed transaction costs. European Journal of Operational Research, 211: 568-576.

[Yin et al., 2010] Yin, G., Jin, Z. and Yang, H. (2010). Asymptotically optimal dividend policy for regimeswitching compound Poisson models Acta Mathematicae Applicatae Sinica (English Series), 26 (4): 529-542.

[Yin et al., 2003] Yin, G., Zhang, Q., Badowski, G. (2003). Discrete-time singularly perturbed Markov chains: Aggregation, occupation measures, and switching diffusion limit. Advances in Applied Probability, 35: 449476.

[Yin and Zhu, 2010] Yin, G. and Zhu, C. (2010). Hybrid Switching Diffusions: Properties and Applications. Springer, New York, 2010. 